\title{
Kelvin-Helmholtz and shear instability of a helical flow around a magnetic flux tube
}

\author{
F. Kolesnikov ${ }^{1}$, M. Bünte ${ }^{2}$, D. Schmitt ${ }^{1}$, and M. Schüssler ${ }^{1}$ \\ 1 Max-Planck-Institut für Sonnensystemforschung ${ }^{\star}$, Max-Planck-Str. 2, 37191 Katlenburg-Lindau, Germany \\ 2 Bodenacherstrasse 33, 8121 Benglen, Switzerland
}

Received 15 January 2004 / Accepted 12 March 2004

\begin{abstract}
Magnetic flux concentrations in the solar (sub)photosphere are surrounded by strong downflows, which come into swirling motion owing to the conservation of angular momentum. While such a whirl flow can stabilize a magnetic flux tube against the MHD fluting instability, it potentially becomes subject to Kelvin-Helmholtz and shear instability near the edge of the flux tube, which may lead to twisting of the magnetic field and perhaps even to the disruption of the magnetic structure. As a first step towards studying the relevance of such instabilities, we investigate the stability of an incompressible flow with longitudinal and azimuthal (whirl) components surrounding a cylinder with a uniform longitudinal magnetic field. We find that a sharp jump of the azimuthal flow component at the cylinder boundary always leads to Kelvin-Helmholtz-type instability for sufficiently small wavelength of the perturbation. On the other hand, a smooth and wide enough transition of the azimuthal velocity towards the surface of the cylinder leads to stable configurations, even for a discontinuous profile of the longitudinal flow.
\end{abstract}

Key words. magnetohydrodynamics: MHD - hydrodynamics - instabilities - Sun: magnetic fields - Sun: photosphere

\section{Introduction}

Concentrated magnetic fields in the solar (sub)photosphere ("flux tubes") are surrounded by strong downflows, which have been inferred from the asymmetry and zero-crossing shifts of observed Stokes $V$-profiles (Grossmann-Doerth et al. 1988, 1989; Bünte et al. 1993b; Grossmann-Doerth et al. 1996; Martinez Pillet et al. 1997; Sigwarth et al. 1999). Such flows are also a persistent feature in numerical simulations of solar magnetic flux concentrations (Deinzer et al. 1984; Nordlund \& Stein 1990; Knölker et al. 1991; Steiner et al. 1998; Stein \& Nordlund 2002; Vögler \& Schüssler 2003). The converging downflows come into rapid swirling motion in order to conserve angular momentum (the "bathtub effect") and, by introducing a pressure gradient to compensate the centrifugal force, provide a mechanism which prevents the flux tubes from being fragmented by the interchange (fluting) instability, which would otherwise disrupt the flux concentrations within about a minute (Schüssler 1984, 1986; Steiner 1990; Bünte et al. 1993a,b).

However, the swirling downflows themselves may give rise to instabilities near the interface between a magnetic flux tube and the surrounding external medium. A sharp interface between two media in relative motion is liable to the Kelvin-Helmholtz instability, which may be modified by

Send offprint requests to: $\mathrm{M}$. Schüssler,

e-mail: msch@linmpi.mpg.de

^ Formerly: Max-Planck-Institut für Aeronomie. surface tension, stratification across the boundary, or the variation of the flow field within a boundary layer of finite width (e.g., Chandrasekhar 1961). In the presence of a magnetic field, Kelvin-Helmholtz instability of a flow parallel to the field lines in an otherwise homogeneous medium sets in if the velocity jump exceeds the Alfvén speed (Rae 1983). The criteria for the Kelvin-Helmholtz instability are modified in the case of a finite transition (shear) layer, which also introduces the possibility of resonant flow instability (e.g., Hollweg et al. 1990). In the planar case, such effects of shear layers have been studied in the context of magnetospheric (Taroyan \& Erdélyi 2002, 2003) and coronal physics (Andries \& Goossens 2001a,b). The cylindrical geometry of a magnetic flux tube introduces a new feature, namely the radial stratification of the swirling external medium due to the centrifugal force. The Rayleigh criterion states that a rotating flow is stable with respect to axisymmetric perturbations if the specific angular momentum of the fluid increases with distance from the axis of rotation. However, in the case of a discontinuous rise of the rotational speed across the surface of the flux tube, the resulting vortex sheet is unstable with respect to non-axisymmetric perturbations (Michalke \& Timme 1967; Rotunno 1978). In the absence of gravity, the unstable perturbations do not vary along the rotation axis (tube axis), so that the longitudinal magnetic field does not affect this instability.

The question arises whether a smooth transition of the angular velocity from zero at the surface of the tube could lead to a stable flow configuration around the flux tube. One could think of such a transition layer as resulting from a preceding 
vortex sheet instability. We have therefore considered a threefold structure with a continuous profile of the rotational speed: zero in the flux tube, a transition layer with constant vorticity, and an external potential vortex (zero vorticity, neutrally stable with respect to the Rayleigh criterion). We allow for different values of the density in the three domains. The velocity component along the flux tube is assumed to be discontinous at the tube surface, so that we consider the least favorable case for stability. For simplicity and to obtain an analytically tractable problem, we restrict ourselves to the case of ideal MHD and incompressible motion in a non-stratified medium. Effects of vertical stratification due to gravity are not expected to be very important during the linear phase of the instability because the shear flow instability of the rotational flow is basically twodimensional (horizontal). The assumption of incompressible perturbations is justified as long as all fluid velocities remain small compared to the sound speed. While this condition is well satisfied in the solar convection zone, the Mach number may occasionally reach values of order unity in the photosphere. The stability properties derived here may thus be modified in the case of high-speed vortices around photospheric flux tubes. In subsequent work, we will therefore extend this study to the case of compressible flows. The stability of systems with a helical flow and a magnetic field is also of considerable interest in connection with astrophysical jets (e.g., Bodo et al. 1989, 1996) and accretion disks (e.g., Ogilvie \& Pringle 1996).

The paper is organized as follows. In Sect. 2, we describe the equilibrium configuration. We derive the dispersion relation from the linearized perturbation equations in Sect. 3. In Sect. 4, we discuss the case of a sharp jump (transition layer of vanishing thickness) and present the results for a finite transition layer in Sect. 5. Our conclusions are given in Sect. 6.

\section{The vortex flow configuration}

We consider an infinite, uniformly magnetized, cylindrical flux tube of radius $R_{0}$. We use cylindrical coordinates $(r, \varphi, z)$ with the axis of the flux tube along the $z$-direction. The magnetic field strength is given by

$\boldsymbol{B}_{0}= \begin{cases}B_{0} \boldsymbol{e}_{z}, & r \leq R_{0} \\ 0, & r>R_{0},\end{cases}$

with $B_{0}=$ const and $\boldsymbol{e}_{z}$ the unit vector in the $z$ direction. We assume an unstratified and incompressible medium, but allow for different values of the density in the cylinder interior, $\rho_{0 \mathrm{i}}$, and exterior, $\rho_{0 \mathrm{e}}$, and in a transition layer $\left(R_{0} \leq r \leq R\right), \rho_{0 \mathrm{t}}$, so that

$\rho_{0}= \begin{cases}\rho_{0 \mathrm{i}}=\eta \rho_{0 \mathrm{e}}, & r \leq R_{0} \\ \rho_{0 \mathrm{t}}=\mu \rho_{0 \mathrm{e}}, & R_{0}<r \leq R \\ \rho_{0 \mathrm{e}}, & r>R,\end{cases}$

with $\eta, \mu$ both constant. The flow around the flux tube has a helical form,

$\boldsymbol{v}_{0}= \begin{cases}0, & r \leq R_{0} \\ r \Omega_{\mathrm{t}}(r) \boldsymbol{e}_{\varphi}+v_{0 z} \boldsymbol{e}_{z}, & R_{0}<r \leq R \\ r \Omega_{\mathrm{e}}(r) \boldsymbol{e}_{\varphi}+v_{0 z} \boldsymbol{e}_{z}, & r>R,\end{cases}$ where $v_{0 z}$ is constant and the angular velocities $\Omega_{\mathrm{e}}$ and $\Omega_{\mathrm{t}}$ depend only on $r$. In an inviscid medium, rotational motion is stable with respect to axisymmetric displacements of fluid elements if the specific angular momentum, $r^{2} \Omega$, increases radially outwards. This is the Rayleigh criterion, which can be written as

$\Phi(r) \equiv \frac{1}{r} \frac{\mathrm{d}}{\mathrm{d} r}\left[r^{2} \Omega(r)\right] \geq 0$

for stability. We consider a flow with constant $\Phi>0$ in the transition layer $\left(R_{0}<r \leq R\right)$ and a marginally stable flow $(\Phi=0)$ in the exterior $(r>R)$, such that the angular velocity (and hence the azimuthal flow speed) is continuous at $r=R_{0}$ and $r=R$ :

$\Omega_{\mathrm{t}}(r)=\frac{\Omega_{0} R^{2}}{R^{2}-R_{0}^{2}}\left[1-\left(\frac{R_{0}}{r}\right)^{2}\right]$,

$\Omega_{\mathrm{e}}(r)=\Omega_{0}\left(\frac{R}{r}\right)^{2}$

This choice leads to a minimal effect of $\Omega_{\mathrm{e}}$ on the stability of the shear layer while the form of $\Omega_{\mathrm{t}}$ permits an analytical solution of the linearized equations in particular cases. The equilibrium pressure in the flux tube is constant, while for the transition layer and the exterior we have

$\nabla p_{0}=-\rho_{0}\left(\boldsymbol{v}_{0} \cdot \nabla\right) \boldsymbol{v}_{0}=\rho_{0} r \Omega^{2} \mathbf{e}_{r}$.

The resulting equilibrium pressure profile is then given by

$p_{0}(r)= \begin{cases}p_{0 \mathrm{i}}=\text { const. }, & r \leq R_{0} \\ p_{0 \mathrm{t}}\left(R_{0}\right)+p_{\mathrm{ct}}(r), & R_{0}<r \leq R \\ p_{0 \mathrm{t}}(R)+p_{\mathrm{ce}}(r), & r>R\end{cases}$

with

$p_{0 \mathrm{t}}\left(R_{0}\right)=p_{0 \mathrm{i}}+\frac{B_{0}^{2}}{8 \pi}$

$p_{\mathrm{ct}}(r)=\frac{1}{2} \frac{\rho_{0 \mathrm{t}} \Omega_{0}^{2} R_{0}^{2} R^{4}}{\left(R^{2}-R_{0}^{2}\right)^{2}}\left[4 \ln \left(\frac{R_{0}}{r}\right)+\left(\frac{r}{R_{0}}\right)^{2}-\left(\frac{R_{0}}{r}\right)^{2}\right]$,

$p_{\mathrm{ce}}(r)=\frac{1}{2} \rho_{0 \mathrm{e}} R^{2} \Omega_{0}^{2}\left[1-\left(\frac{R}{r}\right)^{2}\right]$

Figure 1 shows the equilibrium pressure (dashed line) given by Eq. (8), and the angular velocity (solid line) given by Eqs. (5) and (6).

\section{Perturbation equations}

\subsection{Linearization and mode analysis}

The basic equations describing our problem are the equation of continuity, which in the incompressible case reduces to $\nabla \cdot v=0$, the momentum equation, and the induction equation. 


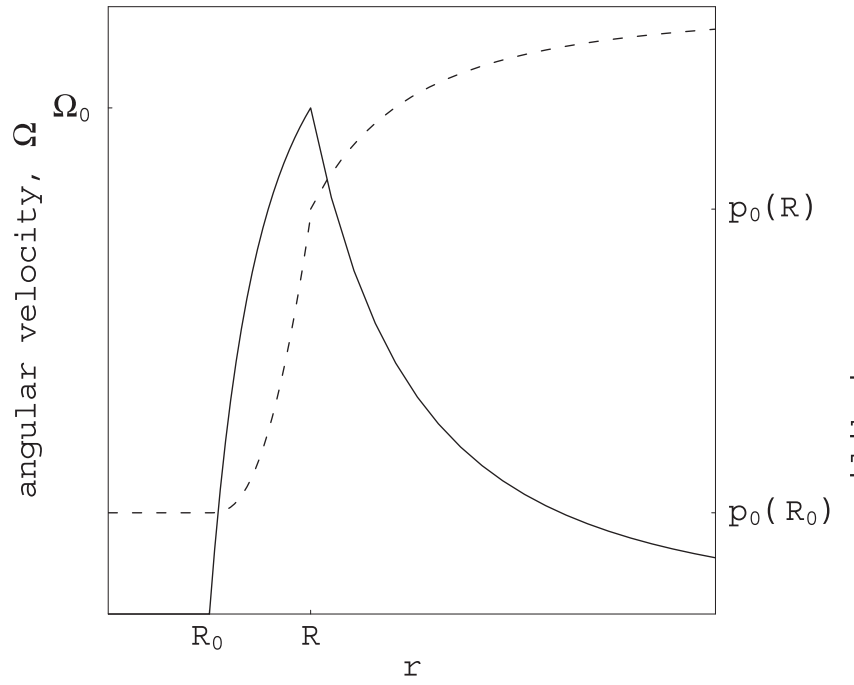

Fig. 1. Profiles of angular velocity (solid line) and pressure (dashed line) for the equilibrium configuration. The magnetic field is restricted to $r \leq R_{0}$.

We linearize these equations about the equilibrium configuration, introducing the first order quantities $p_{1}, \boldsymbol{v}_{1}$, and $\boldsymbol{B}_{1}$ :

$\nabla \cdot \boldsymbol{v}_{1}=0$

$\rho_{0}\left(\partial_{\mathrm{t}}+\boldsymbol{v}_{0} \cdot \nabla\right) \boldsymbol{v}_{1}+\rho_{0}\left(\boldsymbol{v}_{1} \cdot \nabla\right) \boldsymbol{v}_{0}=-\nabla \Pi_{1}+\boldsymbol{T}_{1}$,

$\left(\partial_{\mathrm{t}}+\boldsymbol{v}_{0} \cdot \nabla\right) \boldsymbol{B}_{1}+\left(\boldsymbol{v}_{1} \cdot \nabla\right) \boldsymbol{B}_{0}=\left(\boldsymbol{B}_{0} \cdot \nabla\right) \boldsymbol{v}_{1}+\left(\boldsymbol{B}_{1} \cdot \nabla\right) \boldsymbol{v}_{0}$,

where $\Pi_{1}$ and $\boldsymbol{T}_{1}$ are the perturbations of the total pressure and of the magnetic tension force, respectively:

$\Pi_{1}=p_{1}+\frac{1}{4 \pi} \boldsymbol{B}_{0} \cdot \boldsymbol{B}_{1}$,

$\boldsymbol{T}_{1}=\frac{1}{4 \pi}\left(\boldsymbol{B}_{0} \cdot \nabla\right) \boldsymbol{B}_{1}+\frac{1}{4 \pi}\left(\boldsymbol{B}_{1} \cdot \nabla\right) \boldsymbol{B}_{0}$.

Since equilibrium quantities only depend on $r$, we proceed with a modal analysis and seek solutions whose dependence on $t, \phi$, and $z$ is given by $\exp \left[\mathrm{i}\left(\omega t+m \phi+k_{z} z\right)\right]$, where $\omega$ is the complex frequency, $m$ the azimuthal wave number, and $k_{z}$ the wave number in $z$-direction. This leads to a system of ordinary differential equations for the $r$-dependent perturbation amplitude functions, which can be reduced to a single equation for the amplitude of the radial velocity perturbation, $\hat{v}_{1 r}$ :

$$
\begin{aligned}
\frac{\mathrm{d}^{2} \hat{v}_{1 r}}{\mathrm{~d} r^{2}} & +\left[\frac{1}{r}+\frac{2 m^{2}}{k^{2} r^{3}}+\frac{2 k_{z}^{2} v_{\mathrm{A}}^{2} m}{\left(\sigma^{2}-k_{z}^{2} v_{\mathrm{A}}^{2}\right) r \sigma}(\Phi-2 \Omega)\right] \\
& \times \frac{\mathrm{d} \hat{v}_{1 r}}{\mathrm{~d} r}+\left\{-\left(\frac{1}{r^{2}}-\frac{2 m^{2}}{k^{2} r^{4}}\right)\left[1-\frac{m}{\sigma}\left(\Phi+\frac{2 k_{z}^{2} v_{\mathrm{A}}^{2}}{\sigma^{2}-k_{z}^{2} v_{\mathrm{A}}^{2}} \Omega\right)\right]\right. \\
& +\left[\frac{\sigma^{2}}{\sigma^{2}-k_{z}^{2} v_{\mathrm{A}}^{2}} \frac{2 \Omega}{r}-\frac{\mathrm{d}}{\mathrm{d} r}(\Phi-\Omega)\right] \frac{m}{r \sigma}-k^{2} \\
& +\frac{2 k_{z}^{2} \Omega}{\sigma^{2}-k_{z}^{2} v_{\mathrm{A}}^{2}}\left(\Phi+\frac{2 k_{z}^{2} v_{\mathrm{A}}^{2}}{\sigma^{2}-k_{z}^{2} v_{\mathrm{A}}^{2}} \Omega\right) \\
& \left.-\frac{2 k_{z}^{2} v_{\mathrm{A}}^{2} m^{2}}{\left(\sigma^{2}-k_{z}^{2} v_{\mathrm{A}}^{2}\right) r^{2} \sigma^{2}}(\Phi-2 \Omega)^{2}\right\} \hat{v}_{1 r}=0
\end{aligned}
$$

with

$k^{2}=\frac{m^{2}}{r^{2}}+k_{z}^{2}$

$\sigma(r)=\omega+m \Omega(r)+k_{z} v_{0 z}$

$v_{\mathrm{A}}^{2}=\frac{B_{0}^{2}}{4 \pi \rho_{0}}$.

The expressions for the perturbation amplitudes of the other quantities in terms of $\hat{v}_{1 r}$ are given in Appendix A. Note that Eq. (14) agrees with the incompressible limit of the more general perturbation equations derived by Bondeson et al. (1987) and Goossens et al. (1992).

\subsection{Matching conditions}

Equation (14) can be solved separately in the interior of the cylinder, in the transition layer, and in the exterior. Boundedness of the solutions together with uniqueness of the normal displacement and matching of the total pressure at the perturbed interfaces between interior, transition layer, and exterior, respectively, are required. The (Lagrangian) radial displacement of a mass element is given by

$\delta r=\hat{\delta r} \mathrm{e}^{\mathrm{i}\left(\omega t+m \phi+k_{z} z\right)}$,

where $\hat{\delta r}=\hat{v}_{1 r} / \mathrm{i} \sigma$. At the interfaces, the solutions for $\delta r$ on both sides have to agree in order to have an unique displacement of the interface. This leads to the conditions

$\frac{\hat{v}_{1 r}^{\mathrm{i}}}{\sigma_{\mathrm{i}}}=\frac{\hat{v}_{1 r}^{\mathrm{t}}}{\sigma_{\mathrm{t}}}$ at $r=R_{0}$,

$\frac{\hat{v}_{1 r}^{\mathrm{t}}}{\sigma_{\mathrm{t}}}=\frac{\hat{v}_{1 r}^{\mathrm{e}}}{\sigma_{\mathrm{e}}}$ at $r=R$

where $\sigma_{\mathrm{i}}, \sigma_{\mathrm{t}}$, and $\sigma_{\mathrm{e}}$ are the values of $\sigma$ in the three regions and $\hat{v}_{1 r}^{\mathrm{i}}, \hat{v}_{1 r}^{\mathrm{t}}$, and $\hat{v}_{1 r}^{\mathrm{e}}$ are the corresponding amplitudes of the radial velocity. A further matching condition is the continuity of the normal stress; otherwise, infinite forces would result. In our case, this means that the total (fluid + magnetic) pressure, $\Pi=p+B^{2} / 8 \pi$ has to be continuous. Consequently, we have for the amplitude functions of the total pressure at the perturbed interface between the interior and the transition layer:

$\hat{\Pi}^{\mathrm{i}}\left(R_{0}+\delta r\right)=\hat{\Pi}^{\mathrm{t}}\left(R_{0}+\delta r\right)$.

Expanding this equation to first order in $\delta r$ gives

$\hat{\Pi}_{1}^{\mathrm{i}}\left(R_{0}\right)+\left.\hat{\delta r}\left(R_{0}\right) \frac{\mathrm{d} \hat{\Pi}_{0}^{\mathrm{i}}}{\mathrm{d} r}\right|_{r=R_{0}}=\hat{\Pi}_{1}^{\mathrm{t}}\left(R_{0}\right)+\left.\hat{\delta r}\left(R_{0}\right) \frac{\mathrm{d} \hat{\Pi}_{0}^{\mathrm{t}}}{\mathrm{d} r}\right|_{r=R_{0}}$,

and an analoguous expression for the interface between the transition layer and the exterior. Hence, we obtain the following matching conditions at the interfaces:

$\hat{\Pi}_{1}^{\mathrm{i}}-\hat{\Pi}_{1}^{\mathrm{t}}+\hat{\delta r} \frac{\mathrm{d}}{\mathrm{d} r}\left(\hat{\Pi}_{0}^{\mathrm{i}}-\hat{\Pi}_{0}^{\mathrm{t}}\right)=0$ at $r=R_{0}$,

$\hat{\Pi}_{1}^{\mathrm{t}}-\hat{\Pi}_{1}^{\mathrm{e}}+\hat{\delta} r \frac{\mathrm{d}}{\mathrm{d} r}\left(\hat{\Pi}_{0}^{\mathrm{t}}-\hat{\Pi}_{0}^{\mathrm{e}}\right)=0$ at $r=R$. 
Together with the requirement of boundedness, given by Eqs. (17), (18), (21), and (22), Eq. (14) poses an eigenvalue problem.

\section{Discontinuous vortex flow}

\subsection{Stability criterion}

As a first step, we consider a sharp interface between the cylinder interior and the vortex flow in the exterior, i.e., there is no transition layer $\left(R_{0}=R\right)$ and both the axial and the angular velocity have a jump at $r=R_{0}$ (Bünte 1993a). Under these conditions, Eq. (14) has the same form in the interior $\left(0 \leq r \leq R_{0}\right)$ and the exterior region $\left(R_{0} \leq r\right)$ :

$\frac{\mathrm{d}^{2} \hat{v}_{1 r}}{\mathrm{~d} r^{2}}+\left(\frac{1}{r}+\frac{2 m^{2}}{k^{2} r^{3}}\right) \frac{\mathrm{d} \hat{v}_{1 r}}{\mathrm{~d} r}+\left(-k^{2}-\frac{1}{r^{2}}+\frac{2 m^{2}}{k^{2} r^{4}}\right) \hat{v}_{1 r}=0$.

We obtain bounded solutions in both regions and match them according to the matching conditions. With $x=k_{z} r$ we can write Eq. (23) in the form

$$
\begin{aligned}
x^{2}\left(x^{2}+m^{2}\right)^{2} & \frac{\mathrm{d}^{2} \hat{v}_{1 r}}{\mathrm{~d} x^{2}}+x\left(x^{2}+3 m^{2}\right) \frac{\mathrm{d} \hat{v}_{1 r}}{\mathrm{~d} x} \\
& -\left[\left(x^{2}+m^{2}\right)^{2}+\left(x^{2}-m^{2}\right)\right] \hat{v}_{1 r}=0 .
\end{aligned}
$$

The solution of Eq. (24) is a linear combination of derivatives of the modified Bessel functions of the first and second kind,

$\hat{v}_{1 r}(r)=A_{1} I_{m}^{\prime}\left(k_{z} r\right)+A_{2} K_{m}^{\prime}\left(k_{z} r\right)$,

where $A_{1,2}$ are constants and primes denote derivatives with respect to the argument. The internal solution must be regular at $r=0$, so that $A_{2}=0$. The external solution is bounded only for $A_{1}=0$. Applying the condition of an unique displacement of the interface, Eqs. (17) and (18), and choosing a convenient normalization, the solution is found to be

$\hat{v}_{1 r}(r)=\left\{\begin{array}{l}\sigma_{\mathrm{i}}\left(R_{0}\right) R_{0} \frac{I_{m}^{\prime}\left(k_{z} r\right)}{I_{m}^{\prime}\left(k_{z} R_{0}\right)}, r<R_{0} \\ \sigma_{\mathrm{e}}\left(R_{0}\right) R_{0} \frac{K_{m}^{\prime}\left(k_{z} r\right)}{K_{m}^{\prime}\left(k_{z} R_{0}\right)}, r>R_{0} .\end{array}\right.$

The dispersion relation for the frequency, $\omega$, follows from evaluating the various perturbations in the conditions for matching the total pressure at the interface, Eqs. (21) and (22). This yields the quadratic equation

$$
\begin{aligned}
& (a+\eta b) \omega^{2}+2 a\left(m \Omega_{0}+k_{z} v_{0 z}\right) \omega \\
& \quad+a\left(m \Omega_{0}+k_{z} v_{0 z}\right)^{2}-k_{z} R_{0} \Omega_{0}^{2}-\eta b k_{z}^{2} v_{\mathrm{A}}^{2}=0
\end{aligned}
$$

where $\eta=\rho_{0 \mathrm{i}} / \rho_{0 \mathrm{e}}$ is the constant density ratio and

$$
\begin{aligned}
& a=\frac{K_{m}\left(k_{z} R_{0}\right)}{-K_{m}^{\prime}\left(k_{z} R_{0}\right)}>0, \\
& b=\frac{I_{m}\left(k_{z} R_{0}\right)}{I_{m}^{\prime}\left(k_{z} R_{0}\right)}>0 .
\end{aligned}
$$

In limiting cases we find $a, b \rightarrow k_{z} R_{0} /|m|$ for $|m| \gg k_{z} R_{0}$, and $a, b \rightarrow 1$ for $|m| \ll k_{z} R_{0}$. The system is stable with respect to a given mode $\left(m, k_{z}\right)$ if the determinant of Eq. (27) is nonnegative, i.e. if

$$
\frac{a b \eta}{a+b \eta}\left(m \Omega_{0}+k_{z} v_{0 z}\right)^{2} \leq b \eta k_{z}^{2} v_{\mathrm{A}}^{2}+k_{z} R_{0} \Omega_{0}^{2}
$$

so that $\omega$ is real. If this condition is not met the mode grows at a rate

$$
\begin{aligned}
v\left(m, k_{z}\right)= & \frac{1}{a+b \eta}\left[a b \eta\left(m \Omega_{0}+k_{z} v_{0 z}\right)^{2}\right. \\
& \left.-(a+b \eta)\left(b \eta k_{z}^{2} v_{\mathrm{A}}^{2}+k_{z} R_{0} \Omega_{0}^{2}\right)\right]^{1 / 2} .
\end{aligned}
$$

In the following we discuss two special cases of the stability criterion. First, we consider a purely axial flow $\left(\Omega_{0}=0\right)$, and then the case of a purely azimuthal flow $\left(v_{0 z}=0\right)$.

\subsection{Axial flow}

In the absence of an azimuthal flow component $\left(\Omega_{0}=0\right)$, the stability criterion given by Eq. (29) reduces to

$\frac{a}{a+b \eta} v_{0 z}^{2} \leq v_{\mathrm{A}}^{2}$

We recover the result for the planar case (e.g. Sect. 106 of Chandrasekhar 1961; Rae 1983) in the limit $R_{0} \rightarrow \infty$, in which case $a, b \rightarrow 1$. Figure 2 (left) gives the coefficient of $v_{0 z}^{2}$ in the case $\eta=1$ (i. e. $\rho_{0 \mathrm{i}}=\rho_{0 \mathrm{e}}$ ) as a function of $k_{z} R_{0}$ for different values of $m$. In the planar case $\left(k_{z} R_{0} \gg 1\right)$, the coefficient of $v_{0 z}$ is $(1+\eta)^{-1}=0.5$ (dashed line). For very small and very large wavenumbers $k_{z} R_{0}$ the system behaves as in the planar case. It is slightly more stable for perturbations with intermediate wavenumbers, corresponding to wavelengths of the order of the circumference of the cylinder. Since $a /(a+b \eta) \leq 1 /(1+\eta)$ for all modes $\left(m, k_{z}\right)$, a sufficient condition for stability is $v_{0 z}^{2} \leq v_{\mathrm{A}}^{2}(1+\eta)$. The stability criterion Eq. (31) can be easily generalized to include an internal axial flow and a constant external magnetic field: in the presence of an internal flow, $v_{0 z}$ is replaced by the velocity difference between inside and outside, and in the presence of an external magnetic field the criterion takes the form

$\frac{a b \eta}{a+b \eta} v_{0 z}^{2} \leq a v_{\mathrm{Ae}}^{2}+b \eta v_{\mathrm{Ai}}^{2}$

where $v_{\mathrm{Ai}}$ and $v_{\mathrm{Ae}}$ are the respective internal and external Alfvén speeds. As an example, Fig. 2 (right) shows the Alfvén speed and the (isothermal and adiabatic) sound speeds divided by $\sqrt{1+\eta}$ as a function of height for two empirical solar flux tube models (Solanki 1986) embedded in a non-magnetic atmosphere (Spruit 1977). The figure indicates that the onset of the Kelvin-Helmholtz instability requires an external axial flow velocity well in excess of the sound speed. 

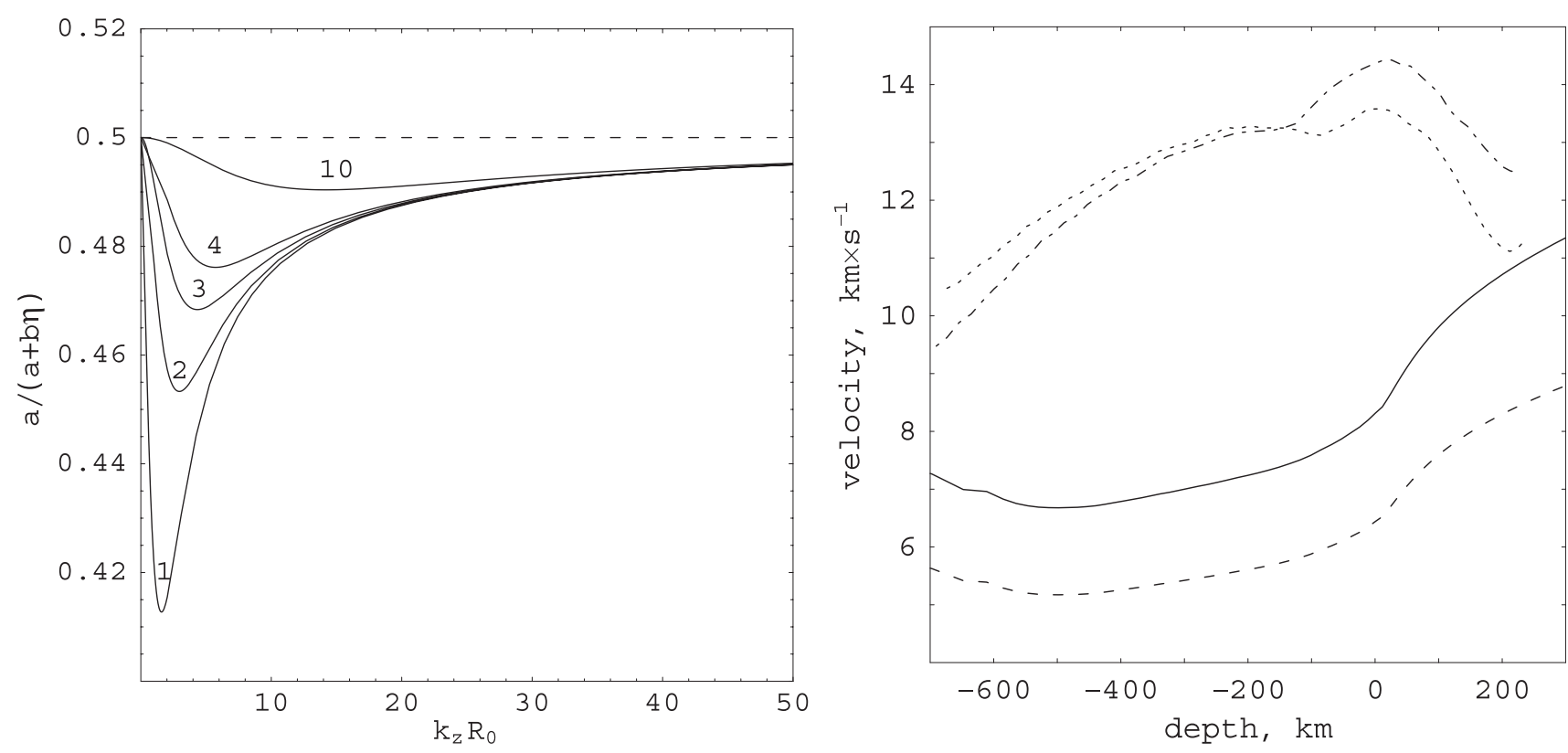

Fig. 2. Stability of a purely axial, discontinuous flow. Left: the coefficient $a /(a+b \eta)$ in Eq. (31) as a function of $k_{z} R_{0}$ in the case $\eta=1$. The various curves correspond to different values of $m$ as indicated; the planar result $\left[a /(a+b \eta) \rightarrow 1 /(1+\eta)=1 / 2\right.$ for $\left.k_{z} R_{0} \rightarrow \infty\right]$ is indicated by the dashed line. Right: shown are Alfvén speeds for the semi-empirical solar flux tube models of Solanki (1986) embedded in non-magnetic atmosphere (Spruit 1977). The dot-dashed line corresponds to the network tube model, while the dotted line represents the plage model. The solid and dashed curves correspond to the adiabatic and isothermal sound speeds, respectively, both divided by $(1+\eta)^{1 / 2}$. All quantities are given as functions of geometrical depth in the solar atmosphere with zero depth corresponding to the level of optical depth unity (visible solar surface).

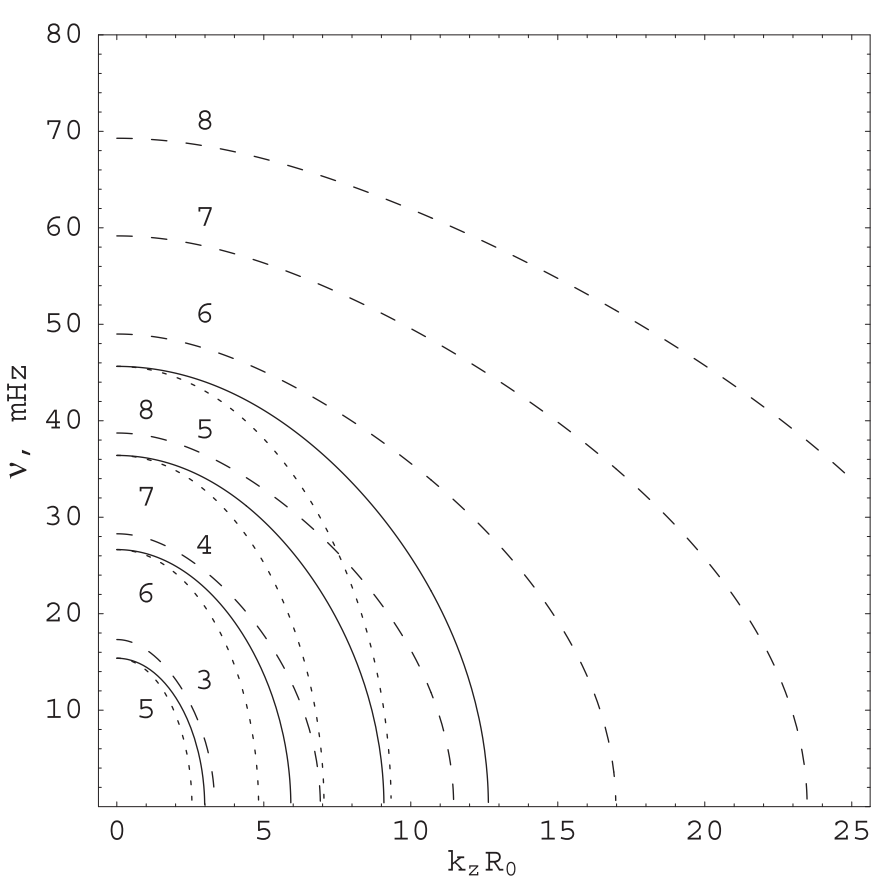

Fig. 3. Growth rates of the Kelvin-Helmholtz instability for a pure external whirl flow of $2 \mathrm{~km} \mathrm{~s}^{-1}$ and a tube radius of $R_{0}=100 \mathrm{~km}$. In the absence of a magnetic field $\left(B_{0}=0\right)$ the dashed lines result for $\eta=1$, the solid lines for $\eta=0.3$. A weak magnetic field of $100 \mathrm{G}$ reduces the growth rates for $k_{z}>0$ as shown by the dotted lines corresponding to the case $\eta=0.3$. The different curves refer to different values of $m$ (numbers on the right refer to the dashed lines, on the left to the solid lines).

\subsection{Rotational flow}

In the case of a pure external whirl flow $\left(v_{0 z}=0\right)$, Eq. (29) indicates that the system is stable if

$\left(\frac{a b \eta}{a+b \eta} \frac{m^{2}}{k_{z} R_{0}}-1\right) \Omega_{0}^{2} R_{0} \leq b \eta k_{z} v_{\mathrm{A}}^{2}$.

Note that $k_{z} \neq 0$ supports the stability of the system by deposition of energy in (a) the magnetic field and (b) in the radial exchange of angular momentum. In Fig. 3 we give the growth rate from Eq. (30) as a function of $k_{z} R_{0}$ for a tube of radius $R_{0}=100 \mathrm{~km}$ surrounded by a whirl flow of $2 \mathrm{~km} \mathrm{~s}^{-1}$. The dashed lines show the result for the non-magnetic case with equal internal and external densities $(\eta=1)$, the solid lines show the case $\eta=0.3$, which appears to be a lower limit for the density contrast of a photospheric magnetic flux tube. The different curves correspond to different azimuthal wavenumbers $m$ as indicated. A weak magnetic field of $100 \mathrm{G}$ reduces the growth rates for $k_{z}>0$ as indicated by the dotted lines for the case $\eta=0.3$. In the limit $k_{z} \rightarrow 0$, we have as stability criterion (for $\Omega_{0} \neq 0$ )

$|m| \leq 1+\frac{1}{\eta}$.

In this limit, the stability criterion depends solely on the azimuthal wavenumber and on the density ratio, but neither on the angular velocity, $\Omega_{0}$, nor on the magnetic field. The system is unstable for all wavenumbers $|m| \geq \bar{m}=1+\eta^{-1}$ with the growth rate

$v(m, 0)=\frac{\Omega_{0}}{1+\eta} \sqrt{\eta|m|(|m|-\bar{m})}$. 
For $\eta=1$ (dashed curves in Fig. 3 ) we have $\bar{m}=2$, for $\eta=0.3$ (solid curves) $\bar{m}=4$. Consequently, a jump in the rotational flow introduces instability for any finite value of $\eta^{-1}$ since (in the absence of viscosity) modes with sufficiently large azimuthal wavenumbers violate the criterion. Since the purely radial modes with $k_{z}=0$ are (most) unstable, the magnetic field cannot stabilize the system.

\section{Finite transition layer}

\subsection{The case $k_{z}=0$}

We now study the stability properties of the helical flow configuration described in Sect. 2. First, we consider the case $k_{z}=0$, i.e., perturbations independent on $z$, which allows an analytical solution. If the perturbations do not vary in the direction along the tube, the magnetic field and the longitudinal flow drop from Eq. (14), which takes an identical form in all three regions:

$\frac{\mathrm{d}^{2} \hat{v}_{1 r}}{\mathrm{~d} r^{2}}+\frac{3}{r} \frac{\mathrm{d} \hat{v}_{1 r}}{\mathrm{~d} r}+\left(\frac{1}{r^{2}}-\frac{m^{2}}{r^{2}}\right) \hat{v}_{1 r}=0$.

The general solution of this equation is given by

$\hat{v}_{1 r}(r)=\alpha_{1}\left(\frac{r}{r_{1}}\right)^{m-1}+\alpha_{2}\left(\frac{r_{2}}{r}\right)^{m+1}$,

where $\alpha_{1,2}$ and $r_{1,2}$ are constants. Boundedness of the internal and the external solutions and applying the condition of uniqueness of the normal displacements leads to the following solutions in the interior $\left(\hat{v}_{1 r}^{\mathrm{i}}\right)$, the transition layer $\left(\hat{v}_{1 r}^{\mathrm{t}}\right)$, and the exterior $\left(\hat{v}_{1 r}^{\mathrm{e}}\right)$ :

$\hat{v}_{1 r}^{\mathrm{i}}(r)=\left[\alpha_{1}+\alpha_{2}\left(\frac{R}{R_{0}}\right)^{m+1}\right]\left(\frac{r}{R_{0}}\right)^{m-1}$,

$\hat{v}_{1 r}^{\mathrm{t}}(r)=\alpha_{1}\left(\frac{r}{R_{0}}\right)^{m-1}+\alpha_{2}\left(\frac{R}{r}\right)^{m+1}$

$\hat{v}_{1 r}^{\mathrm{e}}(r)=\left[\alpha_{1}\left(\frac{R}{R_{0}}\right)^{m-1}+\alpha_{2}\right]\left(\frac{R}{r}\right)^{m+1}$,

for $m \geq 1$. The case $m=0$ is excluded as we consider incompressible flows. For negative $m$, the solutions given by Eqs. (38)-(40) have to be modified accordingly. This leads to identical stability criteria and growth rates independent of the sign $m$ (see Appendix B). We therefore consider only the case of positive $m$ in what follows.

Matching the total pressure at the displaced interface gives a system of two algebraic equations for $\alpha_{1}$ and $\alpha_{2}$. The condition given in Eq. (21) for the total pressure at the interface between the interior and the transition layer takes the form

$\hat{p}_{1}^{\mathrm{i}}\left(R_{0}\right)=\hat{p}_{1}^{\mathrm{t}}\left(R_{0}\right)$,

where $\hat{p}_{1}^{\mathrm{i}}$ and $\hat{p}_{1}^{\mathrm{t}}$ are the amplitudes of the fluid pressure perturbation in the interior of the cylinder and in the transition layer, respectively. This results from $\hat{B}_{1 z}=0$ in the interior (see Eq. (A.6)), the absence of a magnetic field in the transition layer, and from

$\left.\frac{\mathrm{d} \hat{\Pi}_{0}^{\mathrm{i}}}{\mathrm{d} r}\right|_{r=R_{0}}=\left.\frac{\mathrm{d} \hat{\Pi}_{0}^{\mathrm{t}}}{\mathrm{d} r}\right|_{r=R_{0}}=0$ because $\Omega_{\mathrm{i}}\left(R_{0}\right)=\Omega_{\mathrm{t}}\left(R_{0}\right)=0$ and $B_{0}=$ const. Using the relation between $\hat{p}_{1}^{\mathrm{i}}$ and $\hat{p}_{1}^{\mathrm{t}}$ and the corresponding amplitude functions for the radial velocity given by Eq. (A.1), we obtain

$$
\begin{aligned}
\eta\left[\alpha_{1}+\alpha_{2}\left(\frac{R}{R_{0}}\right)^{m+1}\right]= & \mu\left\{\alpha_{1}-\alpha_{2}\left(\frac{R}{R_{0}}\right)^{m+1}\right. \\
& \left.-\left[\alpha_{1}+\alpha_{2}\left(\frac{R}{R_{0}}\right)^{m+1}\right] \frac{\Phi_{\mathrm{t}}}{\omega}\right\},
\end{aligned}
$$

where

$\Phi_{\mathrm{t}}=\frac{1}{r} \frac{\mathrm{d}}{\mathrm{d} r}\left[r^{2} \Omega_{\mathrm{t}}(r)\right]=\frac{2 \Omega_{0} R^{2}}{R^{2}-R_{0}^{2}}$.

From Eq. (42) we obtain

$\left[(\eta-\mu) \omega+\mu \Phi_{\mathrm{t}}\right] \alpha_{1}+\left(\frac{R}{R_{0}}\right)^{m+1}\left[(\eta+\mu) \omega+\mu \Phi_{\mathrm{t}}\right] \alpha_{2}=0$.

The matching condition for the pressure at the interface between transition layer and exterior given by Eq. (22) leads to

$\hat{p}_{1}^{\mathrm{t}}(R)+\left.\frac{\hat{v}_{1 r}^{\mathrm{t}}(R)}{\mathrm{i} \sigma_{\mathrm{t}}(R)} \frac{\mathrm{d} p_{0}^{\mathrm{t}}}{\mathrm{d} r}\right|_{r=R}=\hat{p}_{1}^{\mathrm{e}}(R)+\left.\frac{\hat{v}_{1 r}^{\mathrm{e}}(R)}{\mathrm{i} \sigma_{\mathrm{e}}(R)} \frac{\mathrm{d} p_{0}^{\mathrm{e}}}{\mathrm{d} r}\right|_{r=R}$.

With $\mathrm{d} p_{0}^{\mathrm{t}} / \mathrm{d} r=\rho_{0 b} \Omega_{\mathrm{t}}^{2} r$ and $\mathrm{d} p_{0}^{\mathrm{e}} / \mathrm{d} r=\rho_{0 \mathrm{e}} \Omega_{\mathrm{e}}^{2} r$, and $\sigma_{\mathrm{t}}, \sigma_{\mathrm{e}}, \Omega_{\mathrm{t}}$, $\Omega_{\mathrm{e}}$ given by Eqs. (15), (5) and (6), respectively, we obtain

$$
\begin{aligned}
-\mu\left(\omega+m \Omega_{0}\right) & \frac{R}{m}\left\{\alpha_{1}\left(\frac{R}{R_{0}}\right)^{m-1}-\alpha_{2}\right. \\
- & {\left.\left[\alpha_{1}\left(\frac{R}{R_{0}}\right)^{m-1}+\alpha_{2}\right] \frac{\Phi_{\mathrm{t}}}{\omega+m \Omega_{0}}\right\} } \\
- & {\left[\alpha_{1}\left(\frac{R}{R_{0}}\right)^{m-1}+\alpha_{2}\right] \frac{\mu \Omega_{0}^{2} m}{\omega+m \Omega_{0}} } \\
= & \left(\omega+m \Omega_{0}\right) \frac{R}{m}\left[\alpha_{1}\left(\frac{R}{R_{0}}\right)^{m-1}+\alpha_{2}\right] \\
& -\left[\alpha_{1}\left(\frac{R}{R_{0}}\right)^{m-1}+\alpha_{2}\right] \frac{\Omega_{0}^{2} m}{\omega+m \Omega_{0}} .
\end{aligned}
$$

Hence the second equation for $\alpha_{1}$ and $\alpha_{2}$ follows as

$$
\begin{aligned}
\left(\frac{R}{R_{0}}\right)^{m-1}\left[(\mu+1)\left(\omega+m \Omega_{0}\right)^{2}-\mu \Phi_{\mathrm{t}}\left(\omega+m \Omega_{0}\right)\right. \\
\left.+(\mu-1) \Omega_{0}^{2} m\right] \alpha_{1}+\left[-(\mu-1)\left(\omega+m \Omega_{0}\right)^{2}\right. \\
\left.\quad-\mu \Phi_{\mathrm{t}}\left(\omega+m \Omega_{0}\right)+(\mu-1) \Omega_{0}^{2} m\right] \alpha_{2}=0
\end{aligned}
$$

The dispersion relation is obtained by setting the discriminant of the system of Eqs. (44) and (47) equal to a zero. This results in a cubic equation for the (complex) frequency $\omega$, which we write in the form

$A \beta^{3}+B \beta^{2}+C \beta+D=0$

for the normalized frequency $\beta=\omega / \Omega_{0}$. The expressions for the coefficients of this equation are given in Appendix B. 


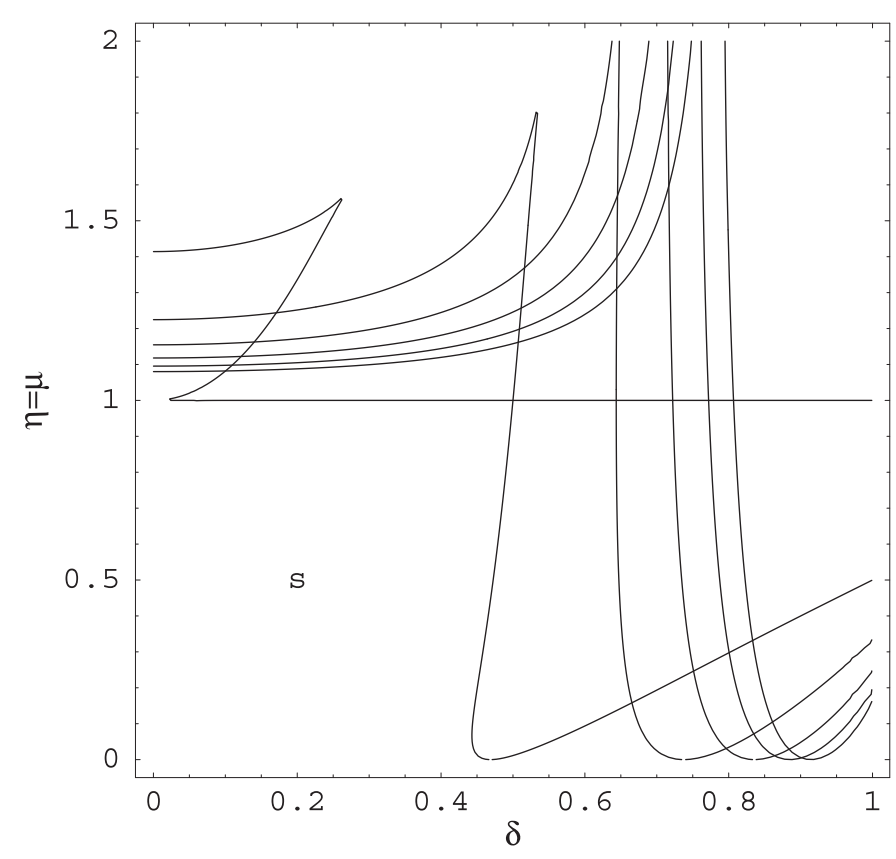

Fig. 4. The lines $Q(\delta, \eta)=0$ in Eq. (49) separating regions of stability and instability for the case $\eta=\mu$ and for the modes with $m=2,3,4,5,6,7$ (from left to right). The region below the line $\eta=1$, left from $m=3$ curve, and below all curves at small values of $\eta=\mu$ (denoted by "s") indicates the domain of stability with respect to perturbations with arbitrary azimuthal wavenumbers.

In the special case $\mu=1$, a common factor $\left(\omega+m \Omega_{0}\right)$ can be separated in the dispersion relation. This represents a (stable) solution $\omega=-m \Omega_{0}$, which corresponds to a stationary wave pattern in the inertial frame. In the general case, the dispersion relation has either three real solutions or one real and two conjugate complex solutions. The flow is unstable in the latter case because then one solution is growing in time. Reducing the cubic equation to an incomplete cubic equation of the form $y^{3}+p y+q=0$, we find that the system is stable provided the condition

$Q=\left(\frac{p}{3}\right)^{3}+\left(\frac{q}{2}\right)^{2} \leq 0$

is fulfilled. The expressions for $p$ and $q$ are given in Appendix $\mathrm{B}^{1}$.

As in the case of a sharp interface and $k_{z}=0$ (Sect. 4.4), the stability criterion does not depend on the value of $\Omega_{0}$; only the growth rates of unstable modes are proportional to $\Omega_{0}$. Figure 4 shows the stability and instability domains in the $(\delta, \eta)$ plane for the case $\eta=\mu$, which means equal density in transition layer and external region. The ratio $\delta=R_{0} / R$ is an inverse measure of the width of the transition layer: $\delta \rightarrow 1$ indicating a sharp transition, $\delta \rightarrow 0$ a very wide transition. Figure 4 shows that for $\eta=\mu<1$ the flow is stable with respect to perturbations with arbitrary azimuthal wavenumber if the width of the transition layer is comparable with the tube radius or larger. The region $\eta=\mu>1$ tends towards instability owing to

${ }^{1}$ For $\eta=\mu=1$ (constant density throughout) we recover the result of Michalke \& Timme (1967) for their case of a stagnant inner cylinder $(\gamma=0)$. the fact that an outward decreasing density corresponds to an effectively Rayleigh-Taylor unstable situation where the centrifugal force plays the role of gravity. The modes with larger $m$ yield larger stability domains. In the case of solar flux tubes, the estimated width of the transition layer is typically $1-10 \mathrm{~km}$ (Schüssler 1986; Hirayama 1992) and the typical flux tube radius is of order of $100 \mathrm{~km}$, so that $\delta \geq 0.9$, well within the unstable region in Fig. 4. The instability of such a rather sharp transition probably leads to the thickening of this layer until it becomes stable for $\delta \lessgtr 0.4$.

In Fig. 5 we give the real (frequency) and imaginary (growth rate) parts of the normalized frequency $\beta=\omega / \Omega_{0}$ as a function of $\eta=\mu$ for $m=2$ and $\delta=0.2$. When two stable modes $\left(\beta_{I}=0\right)$ merge, this creates a conjugate complex pair with the same frequency $\beta_{R}$, one growing $\left(\beta_{I}<0\right)$ and one decaying $\left(\beta_{I}>0\right)$. In the case shown, the combination of three modes leads to the appearance of two unstable domains. For simplicity, we restrict ourselves in this and the following section to showing results only for the case $\eta=\mu$. A strong density reduction in the flux tube would lead to $\eta<\mu$ because of the effect of the magnetic pressure. In view of the outward directed centrifugal force this would increase the stability of the flow (in analogy to a Rayleigh-Taylor stable situation).

\subsection{The general case $\left(k_{z} \neq 0\right)$}

We now allow perturbations which vary along the tube, i.e. $k_{z} \neq 0$. In this case, the effect of both the axial flow $\left(v_{0 z}\right)$ and the magnetic field become relevant. To determine the stability properties we must determine the solutions of Eq. (14) in the interior, in the transition layer, and in the exterior and match them at the interfaces according to Eqs. (17)-(22). In the interior and the exterior, Eq. (14) reduces to Eq. (23). As shown in Sect. 4, bounded solutions in these regions are

$\hat{v}_{1 r}^{\mathrm{i}}(r)=A_{1} I_{m}^{\prime}\left(k_{z} r\right)$

$\hat{v}_{1 r}^{\mathrm{e}}(r)=A_{2} K_{m}^{\prime}\left(k_{z} r\right)$.

In the transition layer, Eq. (14) becomes

$$
\begin{aligned}
\frac{\mathrm{d}^{2} \hat{v}_{1 r}^{\mathrm{t}}}{\mathrm{d} r^{2}} & +\left(\frac{1}{r}+\frac{2 m^{2}}{k^{2} r^{3}}\right) \frac{\mathrm{d} \hat{v}_{1 r}^{\mathrm{t}}}{\mathrm{d} r}+\left[\left(-\frac{1}{r^{2}}+\frac{2 m^{2}}{k^{2} r^{4}}\right)\right. \\
& \left.\times\left(1-\frac{m \Phi_{\mathrm{t}}}{\sigma}\right)-k^{2}+\frac{2 k_{z}^{2}}{\sigma^{2}} \Phi_{\mathrm{t}} \Omega_{\mathrm{t}}+\frac{m \Phi_{\mathrm{t}}}{r^{2} \sigma}\right] \hat{v}_{1 r}^{\mathrm{t}}=0 .
\end{aligned}
$$

Owing to the dependence of $\Omega_{\mathrm{t}}$ on $r$ and the appearance of $\sigma$ in Eq. (52), there is no obvious analytical solution of this equation, so that we resort to a numerical treatment.

Equation (52) has a singular point wherever $\sigma(r)=\omega+$ $m \Omega(r)+k_{z} v_{0 z}=0$. This defines a continuum of real (neutrally stable) modes, $\omega_{\mathrm{c}}$, each of which resonates at a particular radius, $r_{\mathrm{c}}$, where $\omega_{\mathrm{c}}+m \Omega\left(r_{\mathrm{c}}\right)+k_{z} v_{0 z}=0$. Similar effects are known, for example, for Alfvén waves in magnetic flux tubes (e.g., Sakurai et al. 1991; Goossens \& Ruderman 1996). Here we do not consider the continuum of resonant modes, any superposition of which is expected to decay in time by phase mixing. We rather concentrate on the discrete eigenmodes of 

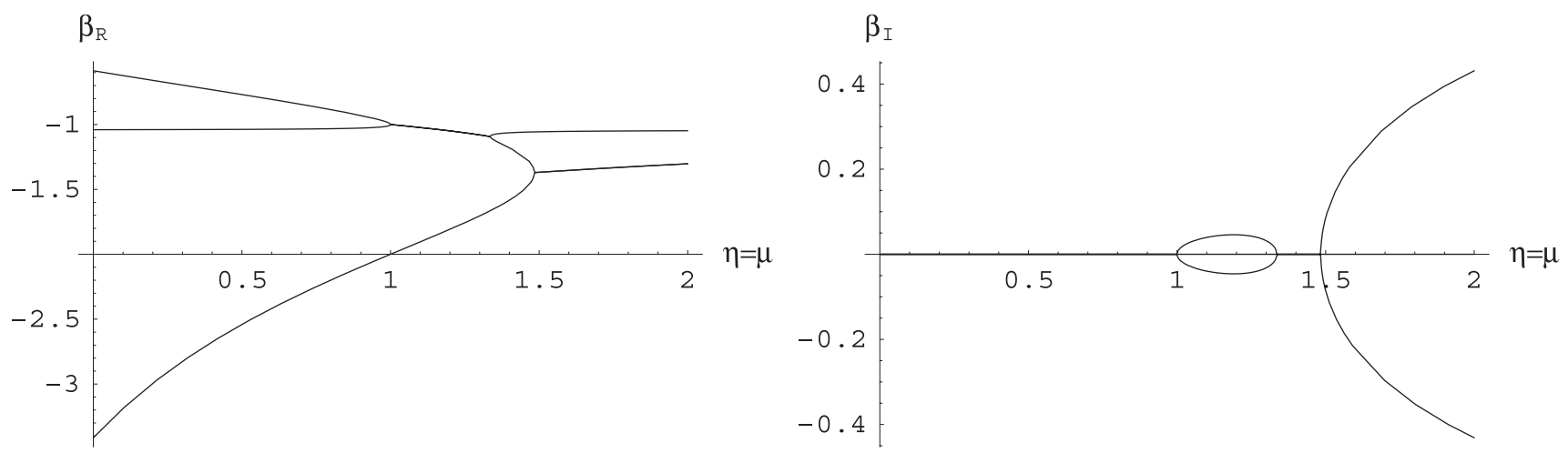

Fig. 5. The mode structure for $m=2, \delta=0.2$ as a function of $\eta(=\mu)$. The real and imaginary parts of $\beta=\omega / \Omega_{0}$ give the frequency and the growth rate of the modes, respectively. Merging of two stable modes leads to the creation of a complex conjugate mode pair, one of which is growing (unstable) and the other decaying.

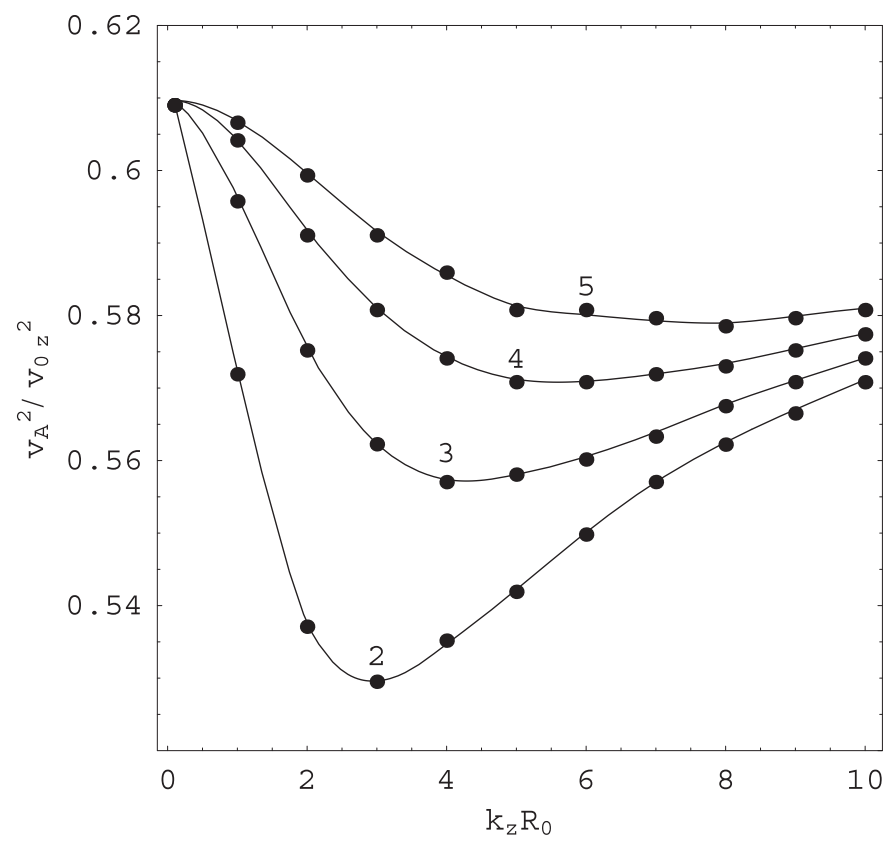

Fig. 6. Comparison between analytical and numerical solutions in the case of a purely longitudinal flow. The curves correspond to neutral stability as given by the analytical result in Eq. (31) for $m=2,3,4$, 5 (from bottom to top). The filled circles give the results for neutral stability based upon the numerical integration and iteration procedure.

the system which describe exponentially growing instabilities. In our numerical treatment of the problem we have therefore avoided the resonant modes. In general, the inclusion of a magnetic field in the transition layer would lead to a continuum of resonant Alfvén waves and, depending on the detailed conditions of the problem, possibly also to discrete overstable quasimodes (Taroyan \& Erdélyi 2002, 2003). Whether such resonant flow instability could also be relevant in the case of vortex flow around magnetic flux tubes will be studied in subsequent work. We solve the boundary value problem for the transition layer by numerical integration of Eq. (52) starting at $r=R_{0}$ and by matching the boundary conditions at the interface between the transition layer and the exterior $(r=R)$ using a shooting method. Details of this procedure are described in Appendix C.
To test the numerical procedure we have considered the case without azimuthal flow. For $\mu=1$, the stability properties of the system with transition layer should then be the same as in the case of a sharp interface discussed in Sect. 4.2. The curves in Fig. 6 show the stability boundaries given by Eq. (31) for various values of $m$. The dots indicate the numerical results obtained with our iteration procedure, which agree fairly well with the analytical values (the maximum deviation is $0.12 \%$ ).

We now consider parameters corresponding to solar magnetic flux tubes, namely $R_{0}=100 \mathrm{~km}, B_{0}=1600 \mathrm{G}, \rho_{\mathrm{i}}=$ $10^{-7} \mathrm{~g} \mathrm{~cm}^{-3}$. The angular velocity $\Omega_{0}=2 \times 10^{-2} \mathrm{~km} \mathrm{~s}^{-1}$ at $r=R$, the velocity component along the tube is $10 \mathrm{~km} \mathrm{~s}^{-1}$. In Figs. $7-10$ we show the resulting stability domains for various azimuthal wavenumbers $(m=2 \ldots 5)$. The dots represent the numerically determined points of neutral stability; they outline the curves separating stable and unstable regions. For each azimuthal wavenumber, the stability domains for $k_{z} R_{0}=10^{-3}$, $10^{-2}, 10^{-1}, 1$ are determined. Perturbations with azimuthal wavenumbers $m=0$ and $m=1$ are always stable. For $k_{z} R_{0}=10^{-3}$, the stability domains for all azimuthal wavenumbers nearly coincide with the stability domains for the case $k_{z}=0$ (compare with Fig. 4). When the perturbation wavelength along the tube decreases $\left(k_{z} R_{0}\right.$ increases $)$, the stability domains for cases with azimuthal wavenumbers $m>2$ grow and the growth rates in the unstable regions decrease. Perturbations with $m=2$ and with values $k_{z} R_{0}$ in the range $0.01 \ldots 0.1$ lead to a somewhat smaller domain of stability: as can be seen in Fig. 7, the stable region is then restricted to smaller values of the density ratios. The shrinking and expanding of the stability domain as a function of $k_{z} R_{0}$ could also be noticed in Fig. 6. Comparing the different modes we find that the stability domain is mostly determined by modes with $m=2$ and 3 . For larger azimuthal wavenumbers, the stability region expands further. Negative $m$ possess the same stability behaviour as their positive counterparts.

We see that the inclusion of variations along the tube $\left(k_{z} \neq 0\right)$ reduces the domain of stable flux tubes by allowing Kelvin-Helmholtz instability of the longitudinal flow. Comparing Fig. 7 with Fig. 4 we find that the domain of stability (with respect to perturbations with arbitrary azimuthal wavenumber $m$ ) has become somewhat smaller, 

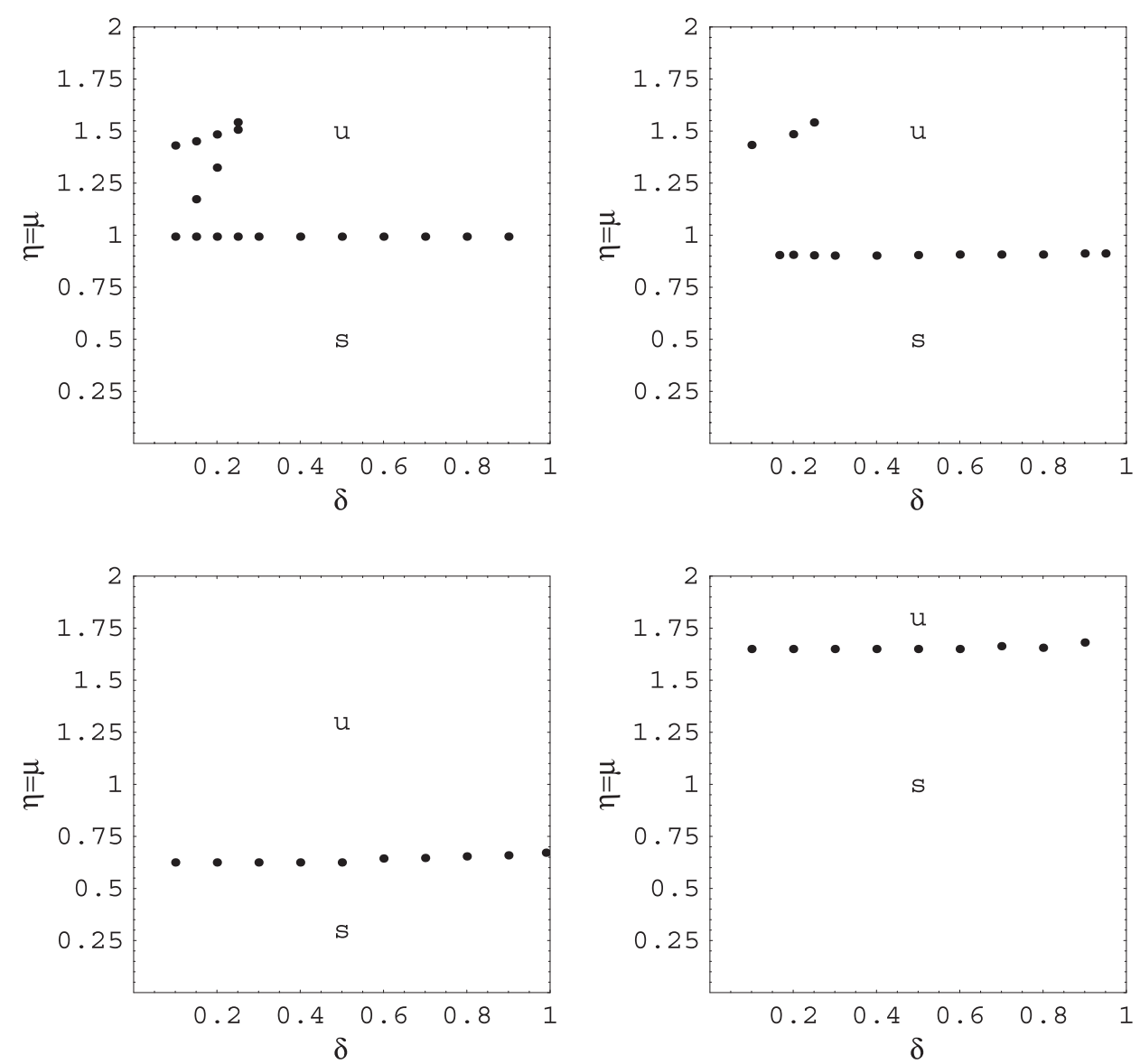

Fig. 7. Domains of stability ("s") and instability ("u") for azimuthal wavenumber $m=2$ and different values of $k_{z} R_{0}$ (upper left: $k_{z} R_{0}=10^{-3}$, upper right: $k_{z} R_{0}=10^{-2}$, lower left: $k_{z} R_{0}=10^{-1}$, lower right: $k_{z} R_{0}=1$ ). The dots indicate the numerically determined points of neutral stability. In the case $k_{z} R_{0}=10^{-3}$, the stability domain is nearly identical to the respective domain for the case $k_{z}=0$ (see Fig. 4). For longer wavelengths of perturbations along the tube the stability domain first shrinks, but reexpands for $k_{z} R_{0}>10^{-1}$. The overall size of the stability domain is mainly limited by perturbations with $k_{z} R_{0} \simeq 10^{-1}$.

( $\eta=\mu \lesssim 0.6, \delta \lessgtr 0.4$ ), mainly with respect to the permitted density ratio. Since solar photospheric flux tubes typically have small density ratios $(\eta \lesssim 0.3)$, this does not affect their stability properties as long as the width of the transition layer is comparable to or larger than the tube radius.

\section{Conclusions}

We have studied the Kelvin-Helmholtz and shear instabilities of a homogeneously magnetized cylinder surrounded by a helical non-magnetic flow in an incompressible and unstratified medium. The azimuthal flow component has a finite transition layer between the stagnant tube interior and the rotating external medium, which is neutrally stable according to the Rayleigh criterion. For perturbations that do not vary along the tube, the magnetic field and the longitudinal flow component do not affect the properties of the shear instability. In this case, a transition layer with a width of the order of the tube radius or larger is required to stabilize the azimuthal flow against perturbations with arbitrary azimuthal wavenumber. For perturbations varying along the tube as well, the Kelvin-Helmholtz instability of the longitudinal flow component leads to a reduction of the stability domain, but flux tubes with sufficiently large internal density deficit and a broad transition layer remain stable with respect to all perturbations. An extended transition layer may naturally evolve as a result of the shear instability of a sharp transition between flux tube and external whirl flow. Consequently, strongly evacuated flux tubes in the solar photosphere surrounded by helical flows may attain stable configurations.

In order to maintain (partial) analytical tractability and simplicity, we could consider only a rather limited class of configuations. This leaves a number of obvious possible (in fact, necessary) extensions of this work, which, however, in most cases require a full numerical treatment. These include the introduction of a finite transition for the vertical flow component, of a (possibly twisted) magnetic field in the transition layer, of gravity, stratification, and compressibility. Among other effects, this would then allow us to study the interchange instability, the resonant flow instability and the magneto-rotational instability (Chandrasekhar 1960; Balbus \& Hawley 1991).

Acknowledgements. We are grateful to an anonymous referee for illuminating remarks and helpful suggestions. 

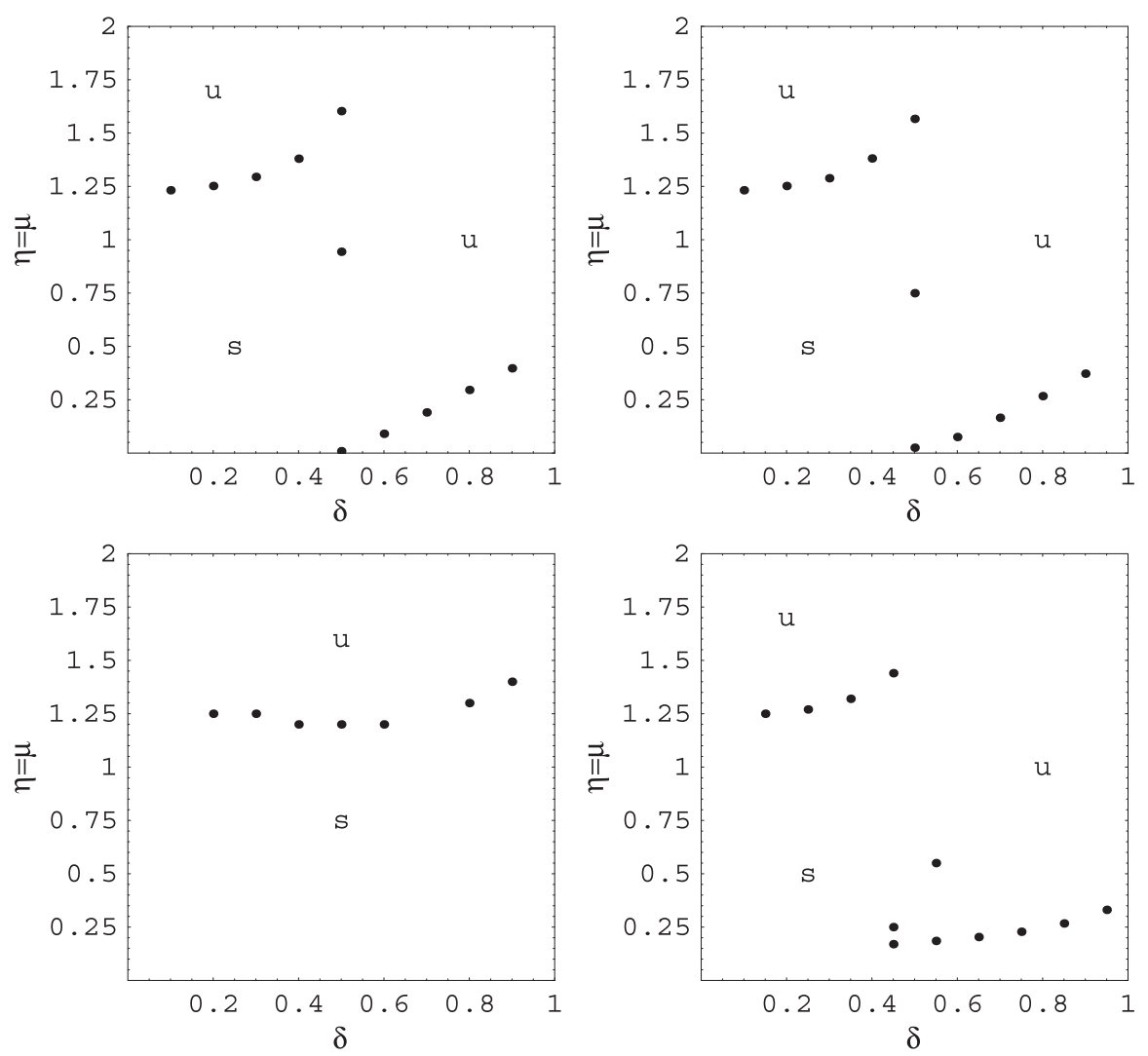

Fig. 8. Same as Fig. 7, but for $m=3$.
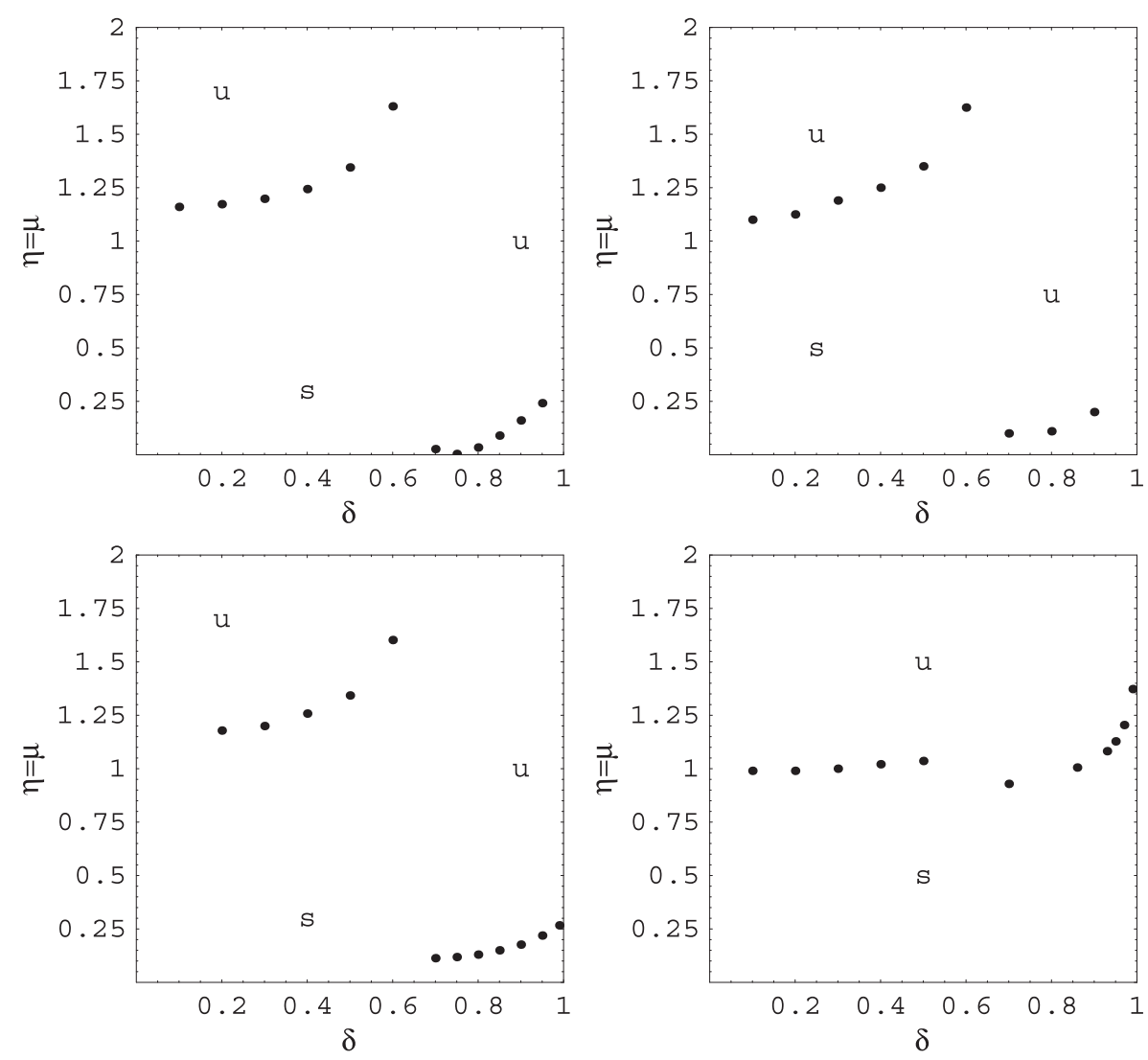

Fig. 9. Same as Fig. 7, but for $m=4$. 

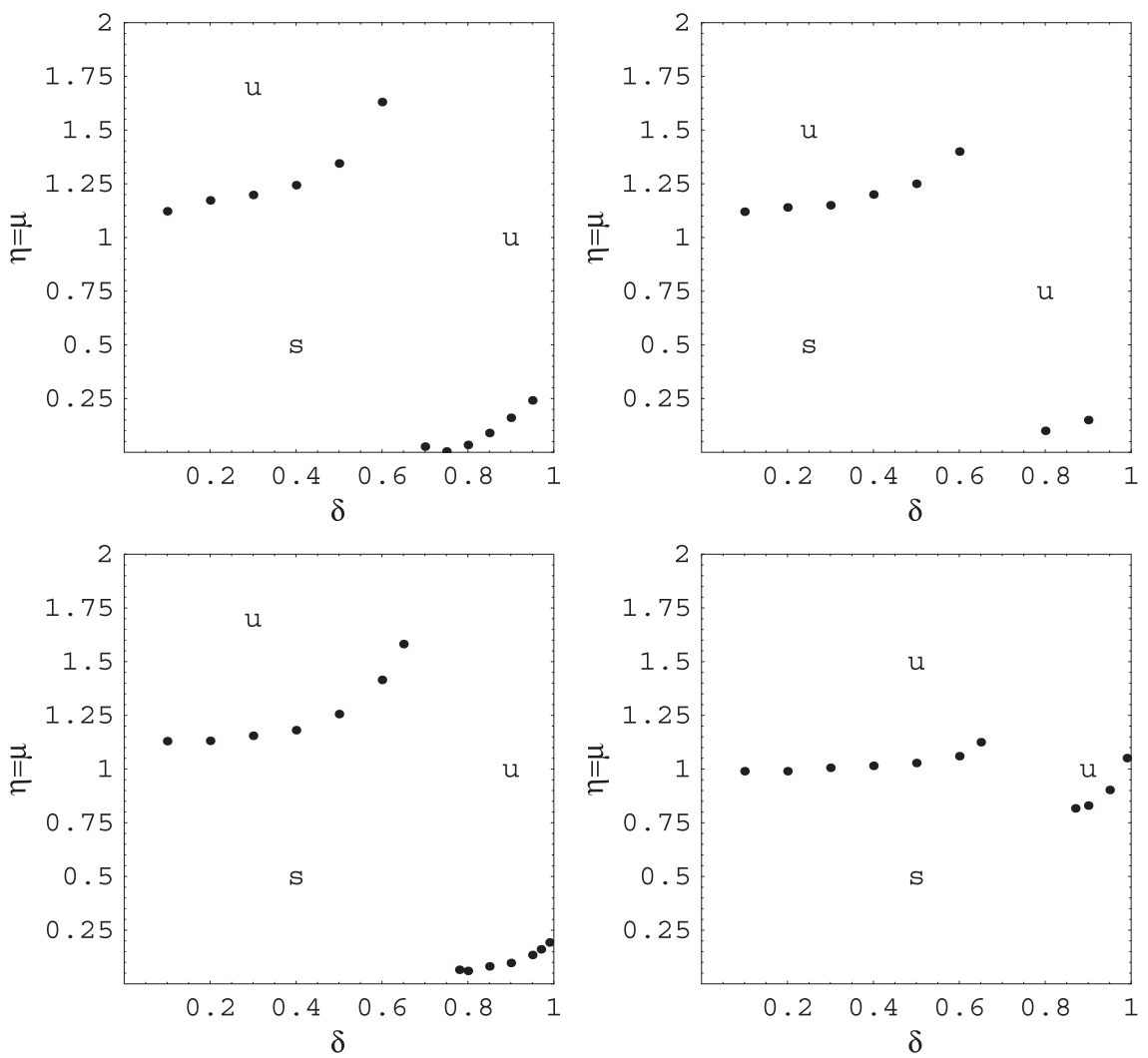

Fig. 10. Same as Fig. 7, but for $m=5$.

\section{Appendix A: Perturbation amplitudes}

The perturbations amplitudes in terms of $\hat{v}_{1 r}$ :

$\hat{p}_{1}=-\mathrm{i} \frac{\rho_{0} \sigma}{k^{2}}\left[D \hat{v}_{1 r}-\Xi \frac{m}{r \sigma} \hat{v}_{1 r}\right]$,

$\hat{v}_{1 \phi}=\frac{\mathrm{i}}{k^{2}}\left[\frac{m}{r} D \hat{v}_{1 r}+\Xi \frac{k_{z}^{2}}{\sigma} \hat{v}_{1 r}\right]$,

$\hat{v}_{1 z}=\frac{\mathrm{i}}{k^{2}}\left[k_{z} D \hat{v}_{1 r}-\Xi \frac{m k_{z}}{r \sigma} \hat{v}_{1 r}\right]$,

$\hat{B}_{1 r}=B_{0} \frac{k_{z}}{\sigma} \hat{v}_{1 r}$,

$\hat{B}_{1 \phi}=\mathrm{i} B_{0} \frac{k_{z}}{\sigma}\left[\frac{m}{r k^{2}} D \hat{v}_{1 r}+\left(\frac{k_{z}^{2}}{\sigma k^{2}} \Xi-\frac{\Phi-2 \Omega}{\sigma}\right) \hat{v}_{1 r}\right]$,

$\hat{B}_{1 z}=\mathrm{i} B_{0} \frac{k_{z}}{\sigma}\left[\frac{k_{z}}{k^{2}} D \hat{v}_{1 r}-\Xi \frac{m k_{z}}{r \sigma k^{2}} \hat{v}_{1 r}\right]$,

where $D \hat{v}_{1 r}$ and $\Xi$ are defined as

$D \hat{v}_{1 r}=\frac{\mathrm{d} \hat{v}_{1 r}}{\mathrm{~d} r}+\frac{\hat{v}_{1 r}}{r}$

$\Xi=\Phi+\frac{2 k_{z}^{2} v_{\mathrm{A}}^{2}}{\sigma^{2}-k_{z}^{2} v_{\mathrm{A}}^{2}} \Omega$.

Appendix B: Coefficients of Eqs. (48) and (49)

For positive $m$ we have

$A=\delta^{2 m}(\eta-\mu)(1-\mu)-(\eta+\mu)(1+\mu)$,
$B=2 m \delta^{2 m}(\eta-\mu)(1-\mu)-2 \mu \frac{\delta^{2 m}}{1-\delta^{2}}(\eta-\mu)+2 \mu \frac{\delta^{2 m}}{1-\delta^{2}}$

$\times(1-\mu)-2 m(\eta+\mu)(1+\mu)+\frac{2 \mu}{1-\delta^{2}}(\eta+\mu)-\frac{2 \mu}{1-\delta^{2}}$

$\times(1+\mu)$,

$C=m^{2} \delta^{2 m}(\eta-\mu)(1-\mu)-2 m \mu \frac{\delta^{2 m}}{1-\delta^{2}}(\eta-\mu)-m \delta^{2 m}$ $\times(\eta-\mu)(1-\mu)+4 m \mu \frac{\delta^{2 m}}{1-\delta^{2}}(1-\mu)-4 \mu^{2} \frac{\delta^{2 m}}{\left(1-\delta^{2}\right)^{2}}$ $-m^{2}(\eta+\mu)(1+\mu)+\frac{2 m \mu}{1-\delta^{2}}(\eta+\mu)+m(\eta+\mu)(1-\mu)$ $-\frac{4 m \mu}{1-\delta^{2}}(1+\mu)+\frac{4 \mu^{2}}{\left(1-\delta^{2}\right)^{2}}$,

$D=2 m^{2} \mu \frac{\delta^{2 m}}{1-\delta^{2}}(1-\mu)-4 m \mu^{2} \frac{\delta^{2 m}}{\left(1-\delta^{2}\right)^{2}}-2 m \mu \frac{\delta^{2 m}}{1-\delta^{2}}$ $\times(1-\mu)-\frac{2 m^{2} \mu}{1-\delta^{2}}(1+\mu)+\frac{4 m \mu^{2}}{\left(1-\delta^{2}\right)^{2}}+\frac{2 m \mu}{1-\delta^{2}}(1-\mu)$, where $\delta=R_{0} / R$.

The parameters of the stability criterion given by Eq. (49) are

$p=-\frac{B^{2}}{3 A^{2}}+\frac{C}{A}$,

$q=\frac{2 B^{3}}{27 A^{3}}-\frac{B C}{3 A^{2}}+\frac{D}{A}$. 
For negative $m$ we have

$A \rightarrow-A, B \rightarrow B, C \rightarrow-C, D \rightarrow D$

This leads to the same stability criteria as for positive $m$.

\section{Appendix C: Procedure for the numerical solution in the case $k_{z} \neq 0$}

The condition for the uniqueness of the normal displacement at the interface given by Eq. (17) and the analytical solution of the equation in the interior given by Eq. (50) yield the initial value of the perturbation amplitude of the radial velocity at $r=R_{0}$ in the transition layer,

$\hat{v}_{1 r}^{\mathrm{t}}\left(R_{0}\right)=\frac{A_{1} I_{m}^{\prime}\left(k_{z} R_{0}\right)\left(\omega+k_{z} v_{0 z}\right)}{\omega}$.

Since $\Omega\left(R_{0}\right)=0$ and $B_{0}=$ const. for $r \leq R_{0}$ we have

$\left.\frac{\mathrm{d}}{\mathrm{d} r} \hat{\Pi}_{0}^{\mathrm{i}}\right|_{r=R_{0}}=\left.\frac{\mathrm{d}}{\mathrm{d} r} \hat{\Pi}_{0}^{\mathrm{t}}\right|_{r=R_{0}}=0$

so that the condition for the total pressure at the interface between the interior and the transition layer, Eq. (21), reduces to

$\hat{\Pi}_{1}^{\mathrm{i}}\left(R_{0}\right)=\hat{\Pi}_{1}^{\mathrm{t}}\left(R_{0}\right)$.

Using Eqs. (1), (12), (15), (A.1), and (A.6), the perturbation of the total pressure in the interior at $r=R_{0}$ takes form

$$
\begin{aligned}
\hat{\Pi}_{1}^{\mathrm{i}}\left(R_{0}\right)= & \hat{p}_{1}^{\mathrm{i}}\left(R_{0}\right)+\frac{1}{4 \pi} B_{0} \hat{B}_{1 z}^{\mathrm{i}}\left(R_{0}\right)=-\mathrm{i} \frac{\rho_{0 \mathrm{i}} \sigma_{\mathrm{i}}}{k^{2}} \\
& \times\left(\left.\frac{\mathrm{d} \hat{v}_{1 r}^{\mathrm{i}}}{\mathrm{d} r}\right|_{r=R_{0}}+\frac{\hat{v}_{1 r}^{\mathrm{i}}}{R_{0}}\right)+\frac{\mathrm{i} k_{z}^{2}}{4 \pi \sigma_{\mathrm{i}} k^{2}} B_{0}^{2}\left(\left.\frac{\mathrm{d} \hat{v}_{1 r}^{\mathrm{i}}}{\mathrm{d} r}\right|_{r=R_{0}}+\frac{\hat{v}_{1 r}^{\mathrm{i}}}{R_{0}}\right) \\
= & \left(-\mathrm{i} \rho_{0 \mathrm{i}} \omega+\frac{\mathrm{i} B_{0}^{2} k_{z}^{2}}{4 \pi \omega}\right)\left(\frac{m^{2}}{R_{0}^{2}}+k_{z}^{2}\right)^{-1} \\
& \times\left(\left.\frac{\mathrm{d} \hat{v}_{1 r}^{\mathrm{i}}}{\mathrm{d} r}\right|_{r=R_{0}}+\frac{\hat{v}_{1 r}^{\mathrm{i}}}{R_{0}}\right) .
\end{aligned}
$$

Similarly, the perturbation of the total pressure in the transition layer at $r=R_{0}$ is obtained as

$$
\begin{aligned}
\hat{\Pi}_{1}^{\mathrm{t}}\left(R_{0}\right)= & \hat{p}_{1}^{\mathrm{t}}\left(R_{0}\right)=-\frac{\mathrm{i} \rho_{0 \mathrm{t}} \sigma_{\mathrm{t}}}{k^{2}}\left(\left.\frac{\mathrm{d} \hat{v}_{1 r}^{\mathrm{t}}}{\mathrm{d} r}\right|_{r=R_{0}}+\frac{\hat{v}_{1 r}^{\mathrm{t}}}{R_{0}}-\frac{\Phi_{\mathrm{t}} m}{R_{0} \sigma_{\mathrm{t}}} \hat{v}_{1 r}^{\mathrm{t}}\right) \\
= & -\mathrm{i} \rho_{0 \mathrm{t}}\left(\omega+k_{z} v_{0 z}\right)\left(\frac{m^{2}}{R_{0}^{2}}+k_{z}^{2}\right)^{-1} \\
& \times\left[\left.\frac{\mathrm{d} \hat{v}_{1 r}^{\mathrm{t}}}{\mathrm{d} r}\right|_{r=R_{0}}+\frac{\hat{v}_{1 r}^{\mathrm{t}}}{R_{0}}\left(1-\frac{\Phi_{\mathrm{t}} m}{\omega+k_{z} v_{0 z}}\right)\right] .
\end{aligned}
$$

Matching the total pressure perturbation at $r=R_{0}$ gives

$$
\begin{aligned}
\frac{\left.\mathrm{d} \hat{v}_{1 r}^{\mathrm{t}}\right|_{r=R_{0}}=}{\mathrm{d} r} & {\left[\mu\left(\omega+k_{z} v_{0 z}\right)\right]^{-1}\left(\eta \omega-\frac{B_{0}^{2} k_{z}^{2}}{4 \pi \omega}\right) } \\
& \times\left(\left.\frac{\mathrm{d} \hat{v}_{1 r}^{\mathrm{i}}}{\mathrm{d} r}\right|_{r=R_{0}}+\frac{\hat{v}_{1 r}^{\mathrm{i}}}{R_{0}}\right)-\left(1-\frac{\Phi_{\mathrm{t}} m}{\omega+k_{z} v_{0 z}}\right) \frac{\hat{v}_{1 r}^{\mathrm{t}}}{R_{0}},
\end{aligned}
$$

where $\hat{v}_{1 r}^{\mathrm{i}}\left(R_{0}\right)$ and $\hat{v}_{1 r}^{\mathrm{t}}\left(R_{0}\right)$ are determined by Eqs. (50) and (C.1). The required derivatives of the analytical solutions in the interior and the exterior are given further.

The numerical integration of Eq. (52) yields the perturbation amplitude of the radial velocity in the transition layer and its first derivative at $r=R$. The matching conditions at the interface between the transition layer and the exterior are only fulfilled for an eigenvalue, $\omega$, of the problem. We iterate $\omega$ with a Newton-Raphson method until we match the condition for the total pressure at the interface. According to Eqs. (18), (51), and (15), the condition for the uniqueness of the normal displacement at $r=R$ is

$$
\frac{\hat{v}_{1 r}^{\mathrm{t}}(R)}{\mathrm{i}\left(\omega+m \Omega_{0}+k_{z} v_{0 z}\right)}=\frac{A_{2} K_{m}^{\prime}\left(k_{z} R\right)}{\mathrm{i}\left(\omega+m \Omega_{0}+k_{z} v_{0 z}\right)} .
$$

From the value of $\hat{v}_{1 r}^{\mathrm{t}}(R)$ obtained as result of the numerical integration we can determine the value of $A_{2}$ :

$A_{2}=\frac{\hat{v}_{1 r}^{\mathrm{t}}}{K_{m}^{\prime}\left(k_{z} R\right)}$.

The condition Eq. (22) for matching the total pressure at $r=R$ is

$\hat{\Pi}_{1}^{\mathrm{t}}(R)+\left.\hat{\delta} r(R) \frac{\mathrm{d}}{\mathrm{d} r} \hat{\Pi}_{0}^{\mathrm{t}}\right|_{r=R}=\hat{\Pi}_{1}^{\mathrm{e}}(R)+\left.\hat{\delta} r(R) \frac{\mathrm{d}}{\mathrm{d} r} \hat{\Pi}_{0}^{\mathrm{e}}\right|_{r=R}$.

Since the magnetic field vanishes in the transition layer and in the exterior we have

$\hat{p}_{1}^{\mathrm{t}}(R)-\hat{p}_{1}^{\mathrm{e}}(R)+\hat{\delta} r(R)\left[\rho_{0 \mathrm{t}} R \Omega_{0}^{2}-\rho_{0 \mathrm{e}} R \Omega_{0}^{2}\right]=0$,

and, with Eq. (7),

$\hat{p}_{1}^{\mathrm{t}}(R)-\hat{p}_{1}^{\mathrm{e}}(R)+\frac{\hat{v}_{1 r}^{\mathrm{t}} R \Omega_{0}^{2}\left(\rho_{0 \mathrm{t}}-\rho_{0 \mathrm{e}}\right)}{i \sigma_{\mathrm{t}}}=0$.

The perturbation amplitude of the pressure in the transition layer for $r=R$ is, according to Eq. (A.1):

$\hat{p}_{1}^{\mathrm{t}}(R)=-\mathrm{i} \frac{\rho_{\mathrm{t}} \sigma_{\mathrm{t}}}{k^{2}}\left[\left.\frac{\mathrm{d} \hat{v}_{1 r}^{\mathrm{t}}}{\mathrm{d} r}\right|_{r=R}+\frac{\hat{v}_{1 r}^{\mathrm{t}}}{R}\left(1-\frac{\Phi_{\mathrm{t}} m}{\sigma_{\mathrm{t}}}\right)\right]$.

Since $\Phi=0$ in the exterior we have for the perturbation amplitude of the pressure in the exterior at $r=R$

$\hat{p}_{1}^{\mathrm{e}}(R)=-\mathrm{i} \frac{\rho_{\mathrm{e}} \sigma_{\mathrm{e}}}{k^{2}}\left(\left.\frac{\mathrm{d} \hat{v}_{1 r}^{\mathrm{e}}}{\mathrm{d} r}\right|_{r=R}+\frac{\hat{v}_{1 r}^{\mathrm{e}}}{R}\right)$.

From Eqs. (C.9)-(C.11) we find that the condition for matching the total pressure at $r=R$ has the form

$$
\begin{aligned}
& \mu\left[\left.\frac{\mathrm{d} \hat{v}_{1 r}^{\mathrm{t}}}{\mathrm{d} r}\right|_{r=R}+\frac{\hat{v}_{1 r}^{\mathrm{t}}}{R}-\frac{\Phi_{\mathrm{t}} m}{R\left(\omega+m \Omega_{0}+k_{z} v_{0 z}\right)} \hat{v}_{1 r}^{\mathrm{t}}\right] \\
& -\left(\left.\frac{\mathrm{d} \hat{v}_{1 r}^{\mathrm{e}}}{\mathrm{d} r}\right|_{r=R}+\frac{\hat{v}_{1 r}^{\mathrm{e}}}{R}\right)+\frac{\hat{v}_{1 r}^{\mathrm{t}} R \Omega_{0}^{2}(\mu-1)\left(\frac{m^{2}}{R^{2}}+k_{z}^{2}\right)}{\left(\omega+m \Omega_{0}+k_{z} v_{0 z}\right)^{2}}=0 .
\end{aligned}
$$

In this condition, $\hat{v}_{1 r}^{\mathrm{t}}$ and its radial derivative results of the numerical integration of Eq. (52) in the transition layer while $\hat{v}_{1 r}^{\mathrm{e}}$ and its radial derivative are determined according to Eq. (51) and Eq. (C.14). Equation (C.12) gives the condition that has to be satisfied by the eigenvalue, $\omega$. 
The required derivatives of the analytical solutions in the interior (see Eq. (50)) are:

$$
\begin{aligned}
& \frac{\mathrm{d} \hat{v}_{1 r}^{\mathrm{i}}}{\mathrm{d} r}=\frac{\mathrm{d}}{\mathrm{d} r}\left[A_{1} I_{m}^{\prime}\left(k_{z} r\right)\right], \\
& I_{m}^{\prime}\left(k_{z} r\right)=\frac{\mathrm{d} I_{m}\left(k_{z} r\right)}{\mathrm{d}\left(k_{z} r\right)}=\frac{I_{m-1}\left(k_{z} r\right)+I_{m+1}\left(k_{z} r\right)}{2}, \\
& \frac{\mathrm{d}}{\mathrm{d} r} I_{m}^{\prime}\left(k_{z} r\right)=\frac{k_{z}}{4}\left[I_{m-2}\left(k_{z} r\right)+2 I_{m}\left(k_{z} r\right)\right. \\
& \left.+I_{m+2}\left(k_{z} r\right)\right] .
\end{aligned}
$$

and in the exterior (see Eq. (51))

$$
\begin{aligned}
\frac{\mathrm{d} \hat{v}_{1 r}^{\mathrm{t}}}{\mathrm{d} r}=\frac{\mathrm{d}}{\mathrm{d} r}\left[A_{2} K_{m}^{\prime}\left(k_{z} r\right)\right] & \\
K_{m}^{\prime}\left(k_{z} r\right)= & \frac{\mathrm{d} K_{m}\left(k_{z} r\right)}{\mathrm{d}\left(k_{z} r\right)}=\frac{K_{m-1}\left(k_{z} r\right)+K_{m+1}\left(k_{z} r\right)}{-2} \\
\frac{\mathrm{d}}{\mathrm{d} r} K_{m}^{\prime}\left(k_{z} r\right)= & \frac{k_{z}}{4}\left[K_{m-2}\left(k_{z} r\right)+2 K_{m}\left(k_{z} r\right)\right. \\
& \left.+K_{m+2}\left(k_{z} r\right)\right] .
\end{aligned}
$$

\section{References}

Andries, J., \& Goossens, M. 2001a, A\&A, 368, 1083 Andries, J., \& Goossens, M. 2001b, A\&A, 375, 1100 Balbus, S. A., \& Hawley, J. F. 1991, ApJ, 376, 214

Bodo, G., Rosner, R., Ferrari, A., \& Knobloch, E. 1989, ApJ, 341, 631 Bodo, G., Rosner, R., Ferrari, A., \& Knobloch, E. 1996, ApJ, 470, 797 Bondeson, A., Iacono, R., \& Bhattacharjee, A. 1987, Phys. Fluids, 30, 2167

Bünte, M. 1993a, Ph.D. Thesis, ETH Zürich, No. 10’357

Bünte, M. 1993b, A\&A, 276, 236

Bünte, M., Steiner, O., \& Pizzo, V. J. 1993a, A\&A, 268, 299

Bünte, M., Solanki, S. K., \& Steiner, O. 1993b, A\&A, 268, 736

Chandrasekhar, S. 1961, Proc. Natl. Acad. Sci., 46, 253

Chandrasekhar, S. 1961, Hydrodynamic and Hydromagnetic Stability (London: Oxford University Press)

Deinzer, W., Hensler, G., Schüssler, M., et al. 1984, A\&A, 139, 426
Goossens, M., Hollweg, J. V., \& Sakurai, T. 1992, Sol. Phys., 138, 233 Goossens, M., \& Ruderman, M. 1996, in Solar and Astrophysical Magnetohydrodynamic Flows, ed. K. C. Tsinganos (Dordrecht: Kluwer), 61

Grossmann-Doerth, U., Schüssler, M., \& Solanki, S. K. 1988, A\&A, 206, L37

Grossmann-Doerth, U., Schüssler, M., \& Solanki, S. K. 1989, A\&A, 221,338

Grossmann-Doerth, U., Keller, C. U., \& Schüssler, M. 1996, A\&A, 315,610

Hirayama, T. 1992, Sol. Phys., 137, 33

Hollweg, J. V., Yang, G., Cadez, V. M., \& Gakovic, B. 1990, ApJ, 349, 335

Knölker, M., Grossmann-Doerth, U., Schüssler, M., et al. 1991, Adv. Space Res., 11, 285

Martinez Pillet, V., Lites, B. W., \& Skumanich, A. 1997, ApJ, 474, 810

Michalke, A., \& Timme, A. 1967, J. Fluid Mech., 29, 647

Nordlund, Å., \& Stein, R. F. 1990, in Solar Photosphere: Structure, Convection and Magnetic Fields, ed. J. O. Stenflo (Dordrecht: Kluwer Academic Publishers), 191

Ogilvie, G. I., \& Pringle, J. E. 1996, MNRAS, 279, 152

Rae, I. C. 1983, A\&A, 126, 209

Rotunno, R. 1978, J. Fluid Mech., 87, 761

Sakurai, T., Goossens, M., \& Hollweg, J. V. 1991, Sol. Phys., 133, 227

Schüssler, M. 1984, A\&A, 140, 453

Schüssler, M. 1986, in Small-scale Magnetic Flux Concentrations in the Solar Photosphere, ed. W. Deinzer, M. Knölker, \& H. H. Voigt, Proceedings of a workshop held in Göttingen (Göttingen: Vandenhoeck \& Ruprecht), 127

Schüssler, M. 1990, in Solar Photosphere: Structure, Convection and Magnetic Fields, ed. J. O. Stenflo (Dordrecht: Kluwer), IAU Symp., 138, 161

Sigwarth, M., Balasubramaniam, K. S., Knölker, M., et al. 1999, A\&A, 349, 941

Solanki, S. K. 1986, A\&A, 168, 311

Spruit, H. C. 1977, Ph.D. Thesis, University of Utrecht, The Netherlands

Stein, R. F., \& Nordlund, ^̊2002, in SOLMAG, Proc. Magnetic Coupling of the Solar Atmosphere Euroconference, ed. H. Sawaya-Lacoste, IAU Colloq., 188, 83

Steiner, O. 1990, Ph.D. Thesis, ETH Zürich, No. 9292

Steiner, O., Grossmann-Doerth, U., Knölker, M., et al. 1998, ApJ, 495, 468

Taroyan, Y., Erdélyi, R. 2002, Phys. Plasmas, 9, 3121

Taroyan, Y., Erdélyi, R. 2003, Phys. Plasmas, 10, 266

Vögler, A., \& Schüssler, M. 2003, Astron. Nahr., 324, 399 